\title{
Synthesis and Anticancer Evaluations of Novel Thiazole Derivatives Derived from 4-Phenylthiazol-2-amine
}

\author{
Amira E. M. Abdallah, ${ }^{1, \star}$ Rafat M. Mohareb, ${ }^{2}$ Maher H. E. Helal ${ }^{1}$ \\ and Germeen J. Mofeed ${ }^{1}$ \\ ${ }^{1}$ Department of Chemistry, Faculty of Science, Helwan University, Ain Helwan, Cairo-11795, A. R. Egypt \\ ${ }^{2}$ Department of Chemistry, Faculty of Science, Cairo University, Giza, A. R. Egypt \\ *Corresponding author: E-mail: amiraelsayed135@yahoo.com
}

Received: $10-12-2020$

\begin{abstract}
Many novel thiazole derivatives were designed and synthesized using 4-phenylthiazol-2-amine. The reactivity of the latter compound toward different chemical reagents was studied. The structure of the newly synthesized compounds was established based on elemental analysis and spectral data. Furthermore, twenty compounds of the synthesized systems were selected and evaluated in $(\mu \mathrm{M})$ as significant anticancer agents towards three human cancer cell lines [MCF-7 (breast adenocarcinoma), NCI-H460 (non-small cell lung cancer), and SF-268 (CNS cancer)] and normal fibroblasts human cell line (WI-38). The results showed that compounds $\mathbf{9}$ and 14a displayed higher effeciency than the reference doxorubicin.
\end{abstract}

Keywords: Anticancer; chromene; 4-phenylthiazol-2-amine; pyridine; pyrimidine; thiophene

\section{Introduction}

Great concern has been recently focused on the development of heterocyclic compound bearing 1,3-thiazole ring system, which has been identified as a central structural element of several biologically active natural products such as thiamine vitamin $\mathrm{B}$, and pharmacologically active substances in a large number of drugs as antibacterial, ${ }^{1,2}$ antifungal, ${ }^{3,4}$ antiviral, ${ }^{5-7}$ anti-inflammatory, ${ }^{8,9}$ anticancer, ${ }^{10-14}$ anti-HIV, ${ }^{15-17}$ anti-oxidant ${ }^{18,19}$ and analgesic drugs. ${ }^{20,21}$ Both classical and non-classical synthetic methods approaches were used to synthesize thiazole derivatives. Some of the examples of such organic synthesis methods were: the reaction between haloketones and thio-amides (Hantzsch thiazole synthesis, 1889), ${ }^{22,23}$ 2-acylamino-ketones reacting with phosphorus pentasulfide (Robinson-Gabriel synthesis), ${ }^{24-26} \alpha$-aminonitrile with carbon disulfide (Cook-Heilbron synthesis), ${ }^{27}$ and the addition of a thiazole anion to an aromatic nitrile, ${ }^{28}$ additionally certain thiazoles can be accessed through the application of the Herz reaction..$^{29}$ Also, various biosynthesis routes lead to the development of the thiazole ring system as required for the formation of thiamine. ${ }^{30}$ Thiazole derivatives were widely used in dyeing, for example, anthroquinone dyes that contain benzothiazole moiety, such as Algol Yellow 8. Also, they were used as non-steroidal anti-inflammatory drugs (NSAID) like Meloxicam (Figure 1), antiretroviral drugs (Ritonavir), antineoplastic drugs (Tiazofurin), antifungal drugs (Abafungin), and antimicrobial drugs (Sulfathiazol). Moreover thiazole derivatives were used as fungicides, such as Thifluzamide, Tricyclazole, and Thiabendazole which were marketed to control various agricultural pests. So far, modifications of the thiazole ring have proven high effectiveness with improved potency and lesser toxicity.

In continuation of our previous work, ${ }^{31-38}$ the current study reported synthesizing some novel thiazole de-



Fig. 1. Meloxicam Structure: 4-hydroxy-2-methyl- $N$-(5-methyl-2thiazolyl)-2H-1,2-benzothiazine-3-carboxamide-1,1-dioxide. 
rivatives based on 4-phenylthiazol-2-amine. The anticancer activity for all the synthesized compounds was evaluated. The latter products have a promising effect, as mentioned earlier by our research groups, in the preparation of a variety of close heterocyclic analogues compounds. ${ }^{39-41}$

\section{Experimental}

\section{1. General}

All melting points were determined on an Electrothermal digital melting point apparatus and are uncorrected. IR spectra ( $\mathrm{KBr}$ discs) were recorded on an FTIR plus 460 or Pye Unicam SP-1000 spectrophotometer (Pye Unicam, UK, Cambridge). ${ }^{1} \mathrm{H}$ NMR spectra were recorded with Varian EM-300 (300 MHz) (Cairo University) instrument in DMSO- $d_{6}$ as solvent using TMS as internal standard, and chemical shifts were expressed as $\delta \mathrm{ppm}$. The mass spectra were recorded with GCMS-QP 1000 Ex Shimadzu (EI, $70 \mathrm{eV}$ ) (Shimadzu, Japan) instrument. Analytical data were obtained from the Micro-analytical Data Unit at Cairo University and were performed on Vario EL III Elemental CHNS analyzer.

\section{2. Chemistry}

\section{2. 1. Synthesis of Ethyl $N$-(4-Phenylthiazol-2-yl) formimidate (1)}

To a solution of 4-phenylthiazol-2-amine (1.76 g, $0.01 \mathrm{~mol})$ in ethanol $(25 \mathrm{~mL})$, triethyl orthoformate $(1.48$ $\mathrm{g}, 0.01 \mathrm{~mol}$ ) was added. The reaction mixture was heated under reflux for three hours, then cooled and neutralized by pouring onto an ice/water mixture containing a few drops of hydrochloric acid. The solid product formed was collected by filtration and crystallized from ethanol.

Dark orange crystals, yield $78 \%$. Mp $235-237^{\circ} \mathrm{C}$. IR $\left(v, \mathrm{~cm}^{-1}\right): 3065-3026(\mathrm{CH}$ aromatic $), 2991,2853(\mathrm{CH}$, $\left.\mathrm{CH}_{2}, \mathrm{CH}_{3}\right), 1644,1485(\mathrm{C}=\mathrm{C}), 1579(\mathrm{C}=\mathrm{N}) .{ }^{1} \mathrm{H}$ NMR $\left(\right.$ DMSO- $\left.d_{6}\right) \delta 1.91\left(\mathrm{t}, J=6.9 \mathrm{~Hz}, 3 \mathrm{H}, \mathrm{CH}_{3}\right), 3.32(\mathrm{q}, J=6.9$ $\left.\mathrm{Hz}, 2 \mathrm{H}, \mathrm{CH}_{2}\right), 7.28(\mathrm{~s}, 1 \mathrm{H}$, thiazole $\mathrm{H}-5), 7.32-7.90(\mathrm{~s}, 1 \mathrm{H}$, $\left.\mathrm{CH} ; \mathrm{m}, 5 \mathrm{H}, \mathrm{C}_{6} \mathrm{H}_{5}\right) .{ }^{13} \mathrm{C}$ NMR (DMSO- $\left.d_{6}\right) \delta 22.5,62.0$, $107.8,127.7$ (2), 128.1, 128.7 (2), 134.3, 148.7, 157.9, 168.6. MS $m / z$ (\%): $234\left[\mathrm{M}^{+}+2\right](0.31), 233\left[\mathrm{M}^{+}+1\right](0.29), 232$ $\left[\mathrm{M}^{+}\right](0.18), 176(100.00), 77\left[\mathrm{C}_{6} \mathrm{H}_{5}\right]^{+}(23.85)$. Anal. Calcd. for $\mathrm{C}_{12} \mathrm{H}_{12} \mathrm{~N}_{2} \mathrm{OS}(232.30)$ : C, 62.04; $\mathrm{H}, 5.21 ; \mathrm{N}, 12.06 ; \mathrm{S}$, 13.80. Found: C, $61.71 ; \mathrm{H}, 4.99 ; \mathrm{N}, 11.66 ; \mathrm{S}, 13.40$.

\section{2. 2. General Procedure for the Synthesis of (4-Phenylthiazol-2-yl) formamidohydrazide Derivatives (2a,b)}

Equimolar amounts of compound 1 (2.32 g, 0.01 $\mathrm{mol})$ and hydrazine $(0.50 \mathrm{~g}, 0.01 \mathrm{~mol})$ or phenylhydrazine $(1.08 \mathrm{~g}, 0.01 \mathrm{~mol})$ in 1,4-dioxane $(25 \mathrm{~mL})$ were heated under reflux for three hours and cooled by pouring onto an ice/water mixture. The solid product formed in each case was collected by filtration, washed with water, and crystallized from 1,4-dioxane.

\section{N"-(4-Phenylthiazol-2-yl)formimidohydrazide (2a)}

Pale yellow crystals, yield $69 \%$. Mp over $300{ }^{\circ} \mathrm{C}$. IR $\left(v, \mathrm{~cm}^{-1}\right): 3413-3168\left(\mathrm{NH}, \mathrm{NH}_{2}\right), 3066(\mathrm{CH}$ aromatic), 2990, $2852(\mathrm{CH}), 1644,1490(\mathrm{C}=\mathrm{C}), 1579(\mathrm{C}=\mathrm{N}) .{ }^{1} \mathrm{H}$ NMR $\left(\right.$ DMSO- $\left.d_{6}\right) \delta 7.32(\mathrm{~s}, 1 \mathrm{H}$, thiazole $\mathrm{H}-5), 7.34-7.90(\mathrm{~s}, 1 \mathrm{H}$, $\left.\mathrm{CH} ; \mathrm{m}, 5 \mathrm{H}, \mathrm{C}_{6} \mathrm{H}_{5}\right), 7.58\left(\mathrm{~s}, 2 \mathrm{H}, \mathrm{NH}_{2}\right), 12.20(\mathrm{~s}, 1 \mathrm{H}, \mathrm{NH})$. MS $m / z$ (\%): $220\left[\mathrm{M}^{+}+2\right]$ (2.06), $219\left[\mathrm{M}^{+}+1\right]$ (4.91), 218 $\left[\mathrm{M}^{+}\right]$(32.03), $176(100.00), 77\left[\mathrm{C}_{6} \mathrm{H}_{5}\right]^{+}(25.03)$. Anal. Calcd. for $\mathrm{C}_{10} \mathrm{H}_{10} \mathrm{~N}_{4} \mathrm{~S}$ (218.28): C, 55.02; H, 4.62; N, 25.67; S, 14.69. Found: C, 55.09; H, 4.39; N, 25.30; S, 14.30 .

\section{$N^{\prime}$-Phenyl- $N$ "'-(4-phenylthiazol-2-yl)formimidohydra- zide (2b)}

Orange crystals, yield $71 \%$. Mp $280-282^{\circ} \mathrm{C}$. IR $(v$, $\left.\mathrm{cm}^{-1}\right)$ : 3426-3168 (2NH), 3066 (CH aromatic), $2991(\mathrm{CH})$, 1643, $1442(\mathrm{C}=\mathrm{C}), 1577(\mathrm{C}=\mathrm{N}) .{ }^{1} \mathrm{H}$ NMR (DMSO- $\left.d_{6}\right) \delta$ $7.32(\mathrm{~s}, 1 \mathrm{H}$, thiazole $\mathrm{H}-5), 7.34-7.90\left(\mathrm{~m}, 10 \mathrm{H}, 2 \mathrm{C}_{6} \mathrm{H}_{5}\right), 7.57$ (s, $1 \mathrm{H}, \mathrm{CH}), 11.40,12.18$ (2s, 2H, 2NH). MS m/z (\%): 293 $\left[\mathrm{M}^{+}-1\right](0.24), 77\left[\mathrm{C}_{6} \mathrm{H}_{5}\right]^{+}(16.04), 69$ (100.00). Anal. Calcd. for $\mathrm{C}_{16} \mathrm{H}_{14} \mathrm{~N}_{4} \mathrm{~S}$ (294.37): C, 65.28; H, 4.79; N, 19.03; S, 10.89. Found: C, $65.48 ; \mathrm{H}, 4.43 ; \mathrm{N}, 18.67 ; \mathrm{S}, 10.53$.

\section{2. 3. Synthesis of $N$-Phenyl- $N^{\prime}$-(4-phenylthiazol- 2-yl)formimidamide (3)}

To a solution of compound $\mathbf{1}(2.32 \mathrm{~g}, 0.01 \mathrm{~mol})$ in 1,4-dioxane $(25 \mathrm{~mL})$, aniline $(0.93 \mathrm{~g}, 0.01 \mathrm{~mol})$ was added. The reaction mixture was heated under reflux for three hours and then cooled by pouring onto an ice/water mixture. The solid product formed was collected by filtration and crystallized from 1,4-dioxane.

Paige crystals, yield $75 \%$. Mp $280-282{ }^{\circ} \mathrm{C}$. IR ( $v$, $\left.\mathrm{cm}^{-1}\right)$ : 3439-3168 (NH), 3066-3027 (CH aromatic), 2991, $2853(\mathrm{CH}), 1644,1492(\mathrm{C}=\mathrm{C}), 1579(\mathrm{C}=\mathrm{N}) .{ }^{1} \mathrm{H}$ NMR (DMSO- $\left.d_{6}\right) \delta 7.29(\mathrm{~s}, 1 \mathrm{H}$, thiazole H-5), 7.32-7.90 (m, $\left.10 \mathrm{H}, 2 \mathrm{C}_{6} \mathrm{H}_{5}\right), 7.55(\mathrm{~s}, 1 \mathrm{H}, \mathrm{CH}), 12.14(\mathrm{~s}, 1 \mathrm{H}, \mathrm{NH}) . \mathrm{MS} m / z$ (\%): $277\left[\mathrm{M}^{+}-2\right]$ (1.68), $77\left[\mathrm{C}_{6} \mathrm{H}_{5}\right]^{+}$(37.99). Anal. Calcd. for $\mathrm{C}_{16} \mathrm{H}_{13} \mathrm{~N}_{3} \mathrm{~S}$ (279.36): C, 68.79; H, 4.69; N, 15.04; $\mathrm{S}$, 11.48. Found: C, 68.48; H, 4.33; N, 15.40; S, 11.88 .

\section{2. 4. Synthesis of 2-((4-Phenylthiazol-2- ylimino)methyl)malononitrile (4) ${ }^{42,43}$}

To a solution of compound $\mathbf{1}(2.32 \mathrm{~g}, 0.01 \mathrm{~mol})$ in 1,4-dioxane $(25 \mathrm{~mL})$ containing a catalytic amount of triethylamine $(0.5 \mathrm{~mL})$, malononitrile $(0.66 \mathrm{~g}, 0.01 \mathrm{~mol})$ was added. The reaction mixture was heated under reflux for three hours, then cooled and neutralized by pouring onto an ice/water mixture containing a few drops of hydrochloric acid. The formed solid product was collected by filtration and crystallized from 1,4-dioxane.

Paige crystals, yield $73 \%$. Mp $203-205{ }^{\circ} \mathrm{C}$. IR ( $v$, $\left.\mathrm{cm}^{-1}\right): 3064$ (CH aromatic), 2988, $2855(2 \mathrm{CH}), 2202,2200$ 
(2CN), 1643, $1442(\mathrm{C}=\mathrm{C}), 1577(\mathrm{C}=\mathrm{N}) .{ }^{1} \mathrm{H}$ NMR (DMSO- $\left.d_{6}\right) \delta 3.57(\mathrm{~s}, 1 \mathrm{H}, \mathrm{CH}), 7.29(\mathrm{~s}, 1 \mathrm{H}$, thiazole $\mathrm{H}-5), 7.31-$ $7.90\left(\mathrm{~m}, 5 \mathrm{H}, \mathrm{C}_{6} \mathrm{H}_{5}\right), 7.55(\mathrm{~s}, 1 \mathrm{H}, \mathrm{CH}) .{ }^{13} \mathrm{C}$ NMR (DMSO- $\left.d_{6}\right) \delta 22.5,107.8,114.1$ (2), 125.6, 127.7 (2), 128.7 (2), 134.3, 148.7, 157.9, 168.6. MS m/z (\%): $254\left[\mathrm{M}^{+}+2\right](0.22)$, $253\left[\mathrm{M}^{+}+1\right]$ (0.16), $252\left[\mathrm{M}^{+}\right]$(0.13), 176 (100.00), 77 $\left[\mathrm{C}_{6} \mathrm{H}_{5}\right]^{+}$(0.35). Anal. Calcd. for $\mathrm{C}_{13} \mathrm{H}_{8} \mathrm{~N}_{4} \mathrm{~S}$ (252.29): C, 61.89; H, 3.20; N, 22.21; S, 12.71. Found: C, 61.77; H, 3.60; $\mathrm{N}, 21.91 ; \mathrm{S}, 12.53$.

\section{2. 5. Synthesis of 1-Phenyl-3-(4-phenylthiazol- 2-yl)thiourea $(5)^{44,45}$}

To a solution of 4-phenylthiazol-2-amine (1.76 g, $0.01 \mathrm{~mol})$ in 1,4-dioxane $(20 \mathrm{~mL})$ containing a catalytic amount of triethylamine $(0.5 \mathrm{~mL})$, phenyl isothiocyanate $(1.35 \mathrm{~g}, 0.01 \mathrm{~mol})$ was added. The reaction mixture was heated under reflux for three hours then poured onto an ice/water mixture containing a few drops of hydrochloric acid. The formed solid product was collected by filtration, dried, and then crystallized from 1,4-dioxane.

Dark brown crystals, yield $64 \%$. Mp $163-165{ }^{\circ} \mathrm{C}$ [Mp (lit.) $\left.{ }^{44} 225^{\circ} \mathrm{C}\right]$. IR $\left(v, \mathrm{~cm}^{-1}\right): 3440-3154(2 \mathrm{NH})$, $3060(\mathrm{CH}$ aromatic), 1600, $1443(\mathrm{C}=\mathrm{C}), 1573(\mathrm{C}=\mathrm{N})$, 1379, 1288 (C=S). ${ }^{1} \mathrm{H}$ NMR (DMSO- $\left.d_{6}\right) \delta 6.86-8.01(\mathrm{~m}$, $10 \mathrm{H}, 2 \mathrm{C}_{6} \mathrm{H}_{5} ; \mathrm{s}, 1 \mathrm{H}$, thiazole $\left.\mathrm{H}-5\right), 11.10,11.86(2 \mathrm{~s}, 2 \mathrm{H}$, $2 \mathrm{NH}$ ). Anal. Calcd. for $\mathrm{C}_{16} \mathrm{H}_{13} \mathrm{~N}_{3} \mathrm{~S}_{2}$ (311.42): C, 61.71; H, 4.21; N, 13.49; S, 20.59. Found: C, 61.31; H, 4.06; N, 13.89; S, 20.20.

\section{2. 6. Synthesis of 2-Chloro- $N$-(4-phenylthiazol- 2-yl)acetamide (6) ${ }^{46-50}$}

To a solution of 4-phenylthiazol-2-amine (1.76 g, $0.01 \mathrm{~mol})$ in 1,4-dioxane $(20 \mathrm{~mL})$, chloroacetylchloride $(1.12 \mathrm{~g}, 0.01 \mathrm{~mol})$ was added. The reaction mixture was heated under reflux for three hours and then poured onto a beaker containing an ice/water mixture. The formed solid product was collected by filtration, dried, and crystallized from 1,4-dioxane.

Light brown crystals, yield 71\%. Mp $157-159^{\circ} \mathrm{C}[\mathrm{Mp}$ (lit. $\left.)^{46} 171-173{ }^{\circ} \mathrm{C}\right]$; IR $\left(v, \mathrm{~cm}^{-1}\right): 3444-3182(\mathrm{NH}), 3070$ (CH aromatic), 2988, $2877\left(\mathrm{CH}_{2}\right), 1758(\mathrm{C}=\mathrm{O}), 1654,1487$ $(\mathrm{C}=\mathrm{C}), 1565(\mathrm{C}=\mathrm{N}) .{ }^{1} \mathrm{H}$ NMR (DMSO- $\left.d_{6}\right) \delta 4.42(\mathrm{~s}, 2 \mathrm{H}$, $\left.\mathrm{CH}_{2}\right), 7.10-7.90\left(\mathrm{~m}, 5 \mathrm{H}, \mathrm{C}_{6} \mathrm{H}_{5}\right), 7.91(\mathrm{~s}, 1 \mathrm{H}$, thiazole $\mathrm{H}-5)$, $12.66(\mathrm{~s}, 1 \mathrm{H}, \mathrm{NH})$. Anal. Calcd. for $\mathrm{C}_{11} \mathrm{H}_{9} \mathrm{ClN}_{2} \mathrm{OS}(252.72)$ : C, 52.28; H, 3.59; N, 11.08: S, 12.69. Found: C, 51.90; H, $3.97 ; \mathrm{N}, 10.72 ; \mathrm{S}, 12.30$.

\section{2. 7. Synthesis of 2-Cyano- $\mathrm{N}$-(4-phenylthiazol- 2-yl)acetamide (7)}

To a solution of 4-phenylthiazol-2-amine (1.76 g, $0.01 \mathrm{~mol})$ in dimethylformamide $(20 \mathrm{~mL})$, ethyl cyanoacetate $(1.13 \mathrm{~g}, 0.01 \mathrm{~mol})$ was added. The reaction mixture was heated under reflux for three hours then poured onto an ice/water mixture. The solid product formed was collected by filtration and crystallized from ethanol.

Dark orange crystals, yield $75 \%$. Mp $109-111^{\circ} \mathrm{C}$. IR $\left(v, \mathrm{~cm}^{-1}\right): 3444-3164(\mathrm{NH}), 3048(\mathrm{CH}$ aromatic $), 2892$ $\left(\mathrm{CH}, \mathrm{CH}_{2}\right), 1687(\mathrm{C}=\mathrm{O}), 1600,1482(\mathrm{C}=\mathrm{C}), 1564(\mathrm{C}=\mathrm{N})$. ${ }^{1} \mathrm{H}$ NMR (DMSO- $\left.d_{6}\right) \delta 4.07\left(\mathrm{~s}, 2 \mathrm{H}, \mathrm{CH}_{2}\right), 7.30-7.90(\mathrm{~m}$, $\left.5 \mathrm{H}, \mathrm{C}_{6} \mathrm{H}_{5}\right), 7.91(\mathrm{~s}, 1 \mathrm{H}$, thiazole $\mathrm{H}-5), 12.39(\mathrm{~s}, 1 \mathrm{H}, \mathrm{NH})$. Anal. Calcd. for $\mathrm{C}_{12} \mathrm{H}_{9} \mathrm{~N}_{3} \mathrm{OS}$ (243.28): C, 59.24; $\mathrm{H}, 3.73 ; \mathrm{N}$, 17.27; S, 13.18. Found: C, 59.64; H, 4.10; N, 16.88; S, 12.79 .

\section{2. 8. Synthesis of 5-Imino-2-phenyl-6-(1- phenylethylidene)-5H-thiazolo $[3,2-a]$ pyrimidin-7(6H)-one $(8)$}

To a compound $7(2.43 \mathrm{~g}, 0.01 \mathrm{~mol})$ in ammonium acetate $(1.00 \mathrm{~g})$, acetophenone $(1.20 \mathrm{~g}, 0.01 \mathrm{~mol})$ was added. The reaction mixture was heated in an oil bath for two hours and then left to cool. The solid product formed when the product was triturated with ethanol was collected by filtration, then dried and crystallized from ethanol.

Dark yellow crystals, yield $73 \%$. Mp $105-107^{\circ} \mathrm{C}$. IR $\left(v, \mathrm{~cm}^{-1}\right): 3431-3113(\mathrm{NH}), 3063(\mathrm{CH}$ aromatic $), 2990$ $\left(\mathrm{CH}_{3}\right), 1644(\mathrm{C}=\mathrm{O}), 1590,1490(\mathrm{C}=\mathrm{C}), 1524(\mathrm{C}=\mathrm{N}) .{ }^{1} \mathrm{H}$ NMR (DMSO- $\left.d_{6}\right) \delta 2.17\left(\mathrm{~s}, 3 \mathrm{H}, \mathrm{CH}_{3}\right), 6.98-7.90(\mathrm{~m}, 10 \mathrm{H}$, $\left.2 \mathrm{C}_{6} \mathrm{H}_{5}\right), 7.94(\mathrm{~s}, 1 \mathrm{H}$, thiazole $\mathrm{H}-5), 12.20(\mathrm{~s}, 1 \mathrm{H}, \mathrm{NH}) .{ }^{13} \mathrm{C}$ NMR (DMSO- $\left.d_{6}\right) \delta 22.5,108.4,126.6(2), 127.2,127.7$, 127.9, 128.1 (2), 128.4 (2), 128.7 (2), 129.1, 134.3, 148.7, 158.0, 159.7, 168.2, 168.6. Anal. Calcd. for $\mathrm{C}_{20} \mathrm{H}_{15} \mathrm{~N}_{3} \mathrm{OS}$ (345.42): C, 69.54; H, 4.38; N, 12.17; S, 9.28. Found: C, $69.15 ; \mathrm{H}, 4.39 ; \mathrm{N}, 12.57 ; \mathrm{S}, 9.60$.

\section{2. 9. Synthesis of Ethyl 2,4-Diamino-5-((4- phenylthiazol-2-yl)carbamoyl)thiophene- 3-carboxylate (9)}

To a solution of compound $7(2.43 \mathrm{~g}, 0.01 \mathrm{~mol})$ in 1,4-dioxane $(20 \mathrm{~mL})$ containing a catalytic amount of triethylamine $(0.50 \mathrm{ml})$ each of elemental sulfur $(0.32 \mathrm{~g}, 0.01$ $\mathrm{mol}$ ) and ethyl cyanoacetate $(1.13 \mathrm{~g}, 0.01 \mathrm{~mol})$ were added. The reaction mixture was heated under reflux for three hours. The solid product formed upon pouring onto an acidified ice/water mixture was collected by filtration and crystallized from 1,4-dioxane.

Dark yellow crystals, yield $63 \%$. Mp $156-158^{\circ} \mathrm{C}$. IR $\left(v, \mathrm{~cm}^{-1}\right): 3427-3164\left(\mathrm{NH}, 2 \mathrm{NH}_{2}\right), 3104-3047(\mathrm{CH}$ aromatic), $2892\left(\mathrm{CH}_{2}, \mathrm{CH}_{3}\right), 1756,1687(2 \mathrm{C}=\mathrm{O}), 1565,1478$ $(\mathrm{C}=\mathrm{C}), 1550(\mathrm{C}=\mathrm{N}) .{ }^{1} \mathrm{H}$ NMR $\left(\mathrm{DMSO}-d_{6}\right) \delta 1.20(\mathrm{t}, J=7.2$ $\left.\mathrm{Hz}, 3 \mathrm{H}, \mathrm{CH}_{3}\right), 4.05\left(\mathrm{q}, J=7.2 \mathrm{~Hz}, 2 \mathrm{H}, \mathrm{CH}_{2}\right), 7.30(\mathrm{~s}, 1 \mathrm{H}$, thiazole $\mathrm{H}-5), 7.32-7.91\left(\mathrm{~m}, 5 \mathrm{H}, \mathrm{C}_{6} \mathrm{H}_{5}\right), 8.52(\mathrm{~s}, 4 \mathrm{H}$, $\left.2 \mathrm{NH}_{2}\right), 12.32(\mathrm{~s}, 1 \mathrm{H}, \mathrm{NH}) .{ }^{13} \mathrm{C}$ NMR (DMSO- $\left.d_{6}\right) \delta 38.7$, 66.0, 108.5, 125.7, 127.9 (2), 128.7 (2), 128.0, 130.1, 133.0, 134.1, 148.9, 156.3, 158.0, 159.7, 160.0. MS $\mathrm{m} / z$ (\%): 388 $\left[\mathrm{M}^{+}\right](0.80), 387\left[\mathrm{M}^{+}-1\right]$ (0.36), $386\left[\mathrm{M}^{+}-2\right](0.28), 134$ (100.00), $77\left[\mathrm{C}_{6} \mathrm{H}_{5}\right]^{+}(21.40)$. Anal. Calcd. for $\mathrm{C}_{17} \mathrm{H}-$ ${ }_{16} \mathrm{~N}_{4} \mathrm{O}_{3} \mathrm{~S}_{2}$ (388.46): C, 52.56; H, 4.15; N, 14.42; $\mathrm{S}, 16.51$. Found: C, 52.96; H, 4.29; N, 14.79; S, 16.91. 


\section{2. 10. Synthesis of 4,6-Diamino-2-oxo-1-(4- phenylthiazol-2-yl)-1,2-dihydropyridine- 3-carbonitrile (10)}

To a solution of compound $7(2.43 \mathrm{~g}, 0.01 \mathrm{~mol})$ in 1,4-dioxane $(25 \mathrm{~mL})$ containing a catalytic amount of triethylamine $(0.50 \mathrm{~mL})$, malononitrile $(0.66 \mathrm{~g}, 0.01 \mathrm{~mol})$ was added. The reaction mixture was heated under reflux for three hours. After cooling, the reaction mixture was acidified by a few drops of hydrochloric acid and the crude product was precipitated, collected by filtration and crystallized from 1,4-dioxane.

Dark brown crystals, yield $78 \%$. Mp $150-152^{\circ} \mathrm{C}$. IR (v, cm $\left.{ }^{-1}\right): 3462-3164\left(2 \mathrm{NH}_{2}\right), 3048(\mathrm{CH}$ aromatic), 2212 $(\mathrm{CN}), 1686(\mathrm{C}=\mathrm{O}), 1563,1482(\mathrm{C}=\mathrm{C}), 1550(\mathrm{C}=\mathrm{N}),{ }^{1} \mathrm{H}$ NMR (DMSO- $\left.d_{6}\right) \delta 4.26(\mathrm{~s}, 1 \mathrm{H}$, pyridinone $\mathrm{H}-5), 7.27$ (s, $1 \mathrm{H}$, thiazole $\mathrm{H}-5), 7.30-7.90\left(\mathrm{~m}, 5 \mathrm{H}, \mathrm{C}_{6} \mathrm{H}_{5}\right), 7.91,8.52(2 \mathrm{~s}$, $4 \mathrm{H}, 2 \mathrm{NH}_{2}$ ). Anal. Calcd. for $\mathrm{C}_{15} \mathrm{H}_{11} \mathrm{~N}_{5} \mathrm{OS}$ (309.35): C, 58.24; H, 3.58; N, 22.64; S, 10.37. Found: C, 58.64; H, 3.97; $\mathrm{N}, 22.24 ; \mathrm{S}, 10.67$.

\section{2. 11. Synthesis of 2-Oxo- $N$-(4-phenylthiazol-2- yl)-2H-chromene-3-carboxamide $(11)^{51,52}$}

To a solution of compound $7(2.43 \mathrm{~g}, 0.01 \mathrm{~mol})$ in 1,4-dioxane $(20 \mathrm{~mL})$ containing piperidine $(0.5 \mathrm{~mL})$, salicylaldehyde $(1.22 \mathrm{~g}, 0.01 \mathrm{~mol})$ was added. The reaction mixture was heated under reflux for three hours then poured onto an ice/water mixture containing a few drops of hydrochloric acid. The formed solid product was collected by filtration then crystallized from 1,4-dioxane.

Yellow crystals, yield $81 \%$. Mp $180-182{ }^{\circ} \mathrm{C}$. IR ( $v$, $\left.\mathrm{cm}^{-1}\right)$ : 3374-3263 (NH), 3107 (CH aromatic), 1711, 1627 $(2 \mathrm{C}=\mathrm{O}), 1600,1490(\mathrm{C}=\mathrm{C}), 1539(\mathrm{C}=\mathrm{N}) .{ }^{1} \mathrm{H}$ NMR (DM$\left.\mathrm{SO}-d_{6}\right) \delta 7.24(\mathrm{~s}, 1 \mathrm{H}$, thiazole $\mathrm{H}-5), 6.84-8.05(\mathrm{~m}, 9 \mathrm{H}$, $\left.\mathrm{C}_{6} \mathrm{H}_{4}, \mathrm{C}_{6} \mathrm{H}_{5}\right), 8.34$ (s, $1 \mathrm{H}$, pyrane $\left.\mathrm{H}-4\right), 12.09$ (s, $\left.1 \mathrm{H}, \mathrm{NH}\right)$. MS m/z (\%): $350\left[\mathrm{M}^{+}+2\right]$ (4.14), $349\left[\mathrm{M}^{+}+1\right]$ (12.86), 348 $\left[\mathrm{M}^{+}\right]$(24.95), 173 (100.00), $77\left[\mathrm{C}_{6} \mathrm{H}_{5}\right]^{+}$(13.56). Anal. Calcd. for $\mathrm{C}_{19} \mathrm{H}_{12} \mathrm{~N}_{2} \mathrm{O}_{3} \mathrm{~S}$ (348.38): C, 65.51; H, 3.47; N, 8.04; S, 9.20. Found: C, 65.11; H, 3.86; N, 7.87; S, 9.52.

\section{2. 12. General Procedure for the Synthesis of $\mathrm{N}$-Cyclopentylidene and $\mathrm{N}$-Cyclohexylidene-4-phenylthiazol- 2-amine (12a,b)}

To a solution of 4-phenylthiazol-2-amine (1.76 g, $0.01 \mathrm{~mol})$ in 1,4-dioxane containing a catalytic amount of piperidine $(0.50 \mathrm{~mL})$, either cyclopentanone $(0.84 \mathrm{~g}, 0.01$ mol) or cyclohexanone $(0.98 \mathrm{~g}, 0.01 \mathrm{~mol})$ was added. The reaction mixture was heated under reflux for two hours then cooled, neutralized by pouring onto an acidified ice/ water mixture, and crystallized from 1,4-dioxane.

\section{$\mathrm{N}$-Cyclopentylidene-4-phenylthiazol-2-amine (12a) \\ Orange crystals, yield $73 \%$. Mp $225-227{ }^{\circ} \mathrm{C}$. IR ( $v$,} $\left.\mathrm{cm}^{-1}\right)$ : 2950, $2806\left(\mathrm{CH}_{2}\right), 1586,1456(\mathrm{C}=\mathrm{C}), 1519(\mathrm{C}=\mathrm{N})$.
${ }^{1} \mathrm{H}$ NMR (DMSO- $\left.d_{6}\right) \delta 1.56-1.67\left(\mathrm{~m}, 4 \mathrm{H}, 2 \mathrm{CH}_{2}\right), 2.41-$ $2.51\left(\mathrm{~m}, 4 \mathrm{H}, 2 \mathrm{CH}_{2}\right), 7.33-7.53\left(\mathrm{~m}, 5 \mathrm{H}, \mathrm{C}_{6} \mathrm{H}_{5}\right), 8.44(\mathrm{~s}, 1 \mathrm{H}$, thiazole H-5). MS $m / z(\%): 243\left[\mathrm{M}^{+}+1\right](0.45), 242\left[\mathrm{M}^{+}\right]$ (0.70), 84 (100.00). Anal. Calcd. for $\mathrm{C}_{14} \mathrm{H}_{14} \mathrm{~N}_{2} \mathrm{~S}$ (242.34): C, 69.39; H, 5.82; N, 11.56; S, 13.23. Found: C, 69.22; H, $5.42 ; \mathrm{N}, 11.17 ; \mathrm{S}, 13.52$.

\section{$\mathrm{N}$-Cyclohexylidene-4-phenylthiazol-2-amine (12b)}

Shiny paige crystals, yield $71 \%$. Mp $208-210{ }^{\circ} \mathrm{C}$. IR $\left(v, \mathrm{~cm}^{-1}\right): 2950,2806\left(\mathrm{CH}_{2}\right), 1628,1455(\mathrm{C}=\mathrm{C}), 1586$ $(\mathrm{C}=\mathrm{N}) .{ }^{1} \mathrm{H}$ NMR $\left(\mathrm{DMSO}-d_{6}\right) \delta 1.52-1.71\left(\mathrm{~m}, 6 \mathrm{H}, 3 \mathrm{CH}_{2}\right)$, 2.49-2.51 (m, 4H, 2CH $\mathrm{CH}_{2}, 7.10-7.80\left(\mathrm{~m}, 5 \mathrm{H}, \mathrm{C}_{6} \mathrm{H}_{5}\right), 8.39$ (s, $1 \mathrm{H}$, thiazole, H-5). MS $m / z(\%): 258\left[\mathrm{M}^{+}+2\right](0.05)$, $257\left[\mathrm{M}^{+}+1\right](0.12), 256\left[\mathrm{M}^{+}\right](0.27), 255\left[\mathrm{M}^{+}-1\right](0.29)$, $254\left[\mathrm{M}^{+}-2\right]$ (0.07), 84 (100.00), $77\left[\mathrm{C}_{6} \mathrm{H}_{5}\right]^{+}$(1.46). Anal. Calcd. for $\mathrm{C}_{15} \mathrm{H}_{16} \mathrm{~N}_{2} \mathrm{~S}$ (256.37): C, 70.27; H, 6.29; N, 10.93; S, 12.51. Found: C, 69.88; H, 5.93; N, 10.90; S, 12.11 .

\section{2. 13. Synthesis of 4-Phenyl- $N-(1-$ phenylethylidene)thiazol-2-amine (13) ${ }^{53}$}

To a solution of 4-phenylthiazol-2-amine (1.76 g, $0.01 \mathrm{~mol})$ in ethanol $(20 \mathrm{~mL})$ containing a catalytic amount of triethylamine $(0.5 \mathrm{~mL})$, acetophenone $(1.20 \mathrm{~g}, 0.01 \mathrm{~mol})$ was added. The reaction mixture was heated under reflux for three hours then poured into a beaker containing an acidified ice/water mixture. The formed solid product was collected by filtration and crystallized from ethanol.

Yellow crystals, yield 69\%. Mp $190-192{ }^{\circ} \mathrm{C}$. IR ( $v$, $\left.\mathrm{cm}^{-1}\right): 3070$ ( $\mathrm{CH}$ aromatic), $2800\left(\mathrm{CH}_{3}\right), 1598,1481$ $(\mathrm{C}=\mathrm{C}), 1523(\mathrm{C}=\mathrm{N}) .{ }^{1} \mathrm{H}$ NMR (DMSO- $\left.d_{6}\right) \delta 1.30(\mathrm{~s}, 3 \mathrm{H}$, $\left.\mathrm{CH}_{3}\right), 7.01-7.80\left(\mathrm{~m}, 10 \mathrm{H}, 2 \mathrm{C}_{6} \mathrm{H}_{5}\right), 7.81(\mathrm{~s}, 1 \mathrm{H}$, thiazole, H-5). ${ }^{13} \mathrm{C}$ NMR (DMSO- $d_{6}$ ) $\delta 38.7,101.5,125.5$ (2), 127.0, 127.1 (2), 128.4 (2), 128.6 (2), 131.0, 134.9, 143.0, 149.8, 165.0, 168.2. MS $m / z(\%): 279\left[\mathrm{M}^{+}+1\right](0.46), 278\left[\mathrm{M}^{+}\right]$ (0.78), $277\left[\mathrm{M}^{+}-1\right]$ (0.81), $276\left[\mathrm{M}^{+}-2\right]$ (0.67), 176 (100.00), $77\left[\mathrm{C}_{6} \mathrm{H}_{5}\right]^{+}$(36.83). Anal. Calcd. for $\mathrm{C}_{17} \mathrm{H}_{14} \mathrm{~N}_{2} \mathrm{~S}$ (278.37): C, 73.35; H, 5.07; N, 10.06; S, 11.52. Found: C, 73.66; H, $5.39 ; \mathrm{N}, 10.46 ; \mathrm{S}, 11.90$.

\section{2. 14. General Procedure for the Synthesis of Thiazolo[3,2-a]pyrimidine-6-carbonitrile Derivatives 14a-f}

To a solution of 4-phenylthiazol-2-amine (1.76 g, $0.01 \mathrm{~mol})$ in ethanol $(20 \mathrm{~mL})$ containing a catalytic amount of triethylamine $(0.50 \mathrm{~mL})$, each of either benzaldehyde (1.06 g, $0.01 \mathrm{~mol})$, para-methoxybenzaldehyde (1.08 g, $0.01 \mathrm{~mol})$ or para-chlorobenzaldehyde $(1.12 \mathrm{~g}, 0.01 \mathrm{~mol})$ and either malononitrile $(0.66 \mathrm{~g}, 0.01 \mathrm{~mol})$ or ethyl cyanoacetate $(1.13 \mathrm{~g}, 0.01 \mathrm{~mol})$ were added. The reaction mixture was heated under reflux for six hours and then poured onto an acidified ice/water mixture. The formed solid product was collected by filtration and crystallized from ethanol. 
5-Amino-3,7-diphenyl-8a $H$-thiazolo $[3,2-a]$ pyrimidine-6-carbonitrile (14a)

Off white crystals, yield $75 \%$. Mp $225-227^{\circ} \mathrm{C}$. IR ( $v$, $\left.\mathrm{cm}^{-1}\right)$ : $3419\left(\mathrm{NH}_{2}\right), 3030$ (CH aromatic), $2221(\mathrm{CN}), 1585$, $1448(\mathrm{C}=\mathrm{C}), 1520(\mathrm{C}=\mathrm{N}) .{ }^{1} \mathrm{H}$ NMR (DMSO- $\left.d_{6}\right) \delta 7.59$, $7.60(2 \mathrm{~s}, 2 \mathrm{H}$, thiazole $\mathrm{H}-2$, pyrimidine $\mathrm{H}-8 \mathrm{a}), 7.62-7.98$ $\left(\mathrm{m}, 10 \mathrm{H}, 2 \mathrm{C}_{6} \mathrm{H}_{5}\right), 8.53$ (s, $\left.2 \mathrm{H}, \mathrm{NH}_{2}\right) .{ }^{13} \mathrm{C}$ NMR (DMSO- $d_{6}$ ) $\delta 40.3,81.6,113.2,114.2,127.5,128.0$ (2), 129.6 (2), 129.5 (2), 130.5 (2), 131.3 (2), 134.4, 156.0, 161.5, 162.0. MS $m / z$ (\%): $331\left[\mathrm{M}^{+}+1\right]$ (32.57), 64 (100.00). Anal. Calcd. for $\mathrm{C}_{19} \mathrm{H}_{14} \mathrm{~N}_{4} \mathrm{~S}$ (330.41): C, 69.07; H, 4.27; N, 16.96; S, 9.70. Found: C, 69.39; H, 4.30; N, 16.62; S, 9.31.

5-Amino-7-(4-methoxyphenyl)-3-phenyl-8aH-thiazolo[3,2-a]pyrimidine-6-carbonitrile (14b)

Yellow needles crystals, yield 78\%. Mp $130-132{ }^{\circ} \mathrm{C}$. IR $\left(v, \mathrm{~cm}^{-1}\right)$ : 3406-3283 $\left(\mathrm{NH}_{2}\right), 3114-3025$ ( $\mathrm{CH}$ aromatic), 2978, $2846\left(\mathrm{CH}_{3}\right), 2216(\mathrm{CN}), 1606,1506(\mathrm{C}=\mathrm{C}), 1564$ $(\mathrm{C}=\mathrm{N}) .{ }^{1} \mathrm{H}$ NMR (DMSO- $\left.d_{6}\right) \delta 3.87\left(\mathrm{~s}, 3 \mathrm{H}, \mathrm{CH}_{3}\right), 6.92$, $6.94(2 \mathrm{~s}, 2 \mathrm{H}$, thiazole $\mathrm{H}-2$, pyrimidine $\mathrm{H}-8 \mathrm{a}), 7.11-8.01$ (m, 9H, $\left.\mathrm{C}_{6} \mathrm{H}_{4}, \mathrm{C}_{6} \mathrm{H}_{5}\right), 8.38$ (s, 2H, $\mathrm{NH}_{2}$ ). MS m/z (\%): 361 $\left[\mathrm{M}^{+}+1\right](0.57), 360\left[\mathrm{M}^{+}\right](0.45), 358\left[\mathrm{M}^{+}-2\right](0.45), 134$ (100.00), $77\left[\mathrm{C}_{6} \mathrm{H}_{5}\right]^{+}$(58.08). Anal. Calcd. for $\mathrm{C}_{20} \mathrm{H}_{16} \mathrm{~N}_{4} \mathrm{OS}$ (360.43): C, 66.65; H, 4.47; N, 15.54; S, 8.90. Found: C, $66.81 ; \mathrm{H}, 4.87 ; \mathrm{N}, 15.39 ; \mathrm{S}, 9.22$.

5-Amino-7-(4-chlorophenyl)-3-phenyl-8aH-thiazolo[3,2-a]pyrimidine-6-carbonitrile (14c)

Yellow needles crystals, yield $78 \%$. Mp $228-230{ }^{\circ} \mathrm{C}$. IR $\left(v, \mathrm{~cm}^{-1}\right): 3240\left(\mathrm{NH}_{2}\right), 3092(\mathrm{CH}$ aromatic $), 2223$ $(\mathrm{CN}), 1631,1483(\mathrm{C}=\mathrm{C}), 1581(\mathrm{C}=\mathrm{N}) .{ }^{1} \mathrm{H}$ NMR (DM$\left.\mathrm{SO}-d_{6}\right) \delta 7.21,7.22(2 \mathrm{~s}, 2 \mathrm{H}$, thiazole $\mathrm{H}-2$, pyrimidine $\mathrm{H}-8 \mathrm{a})$, 7.27-7.98 ( $\mathrm{m}, 9 \mathrm{H}, \mathrm{C}_{6} \mathrm{H}_{4}, \mathrm{C}_{6} \mathrm{H}_{5}$ ), 8.52 (s, 2H, $\mathrm{NH}_{2}$ ). Anal. Calcd. for $\mathrm{C}_{19} \mathrm{H}_{13} \mathrm{ClN}_{4} \mathrm{~S}$ (364.85): C, 62.55; H, 3.59; $\mathrm{N}, 15.36 ; \mathrm{S}, 8.79$. Found: C, 62.22; H, 3.21; N, 14.96; S, 9.12 .

5-Hydroxy-3,7-diphenyl-8aH-thiazolo[3,2-a]pyrimidine-6-carbonitrile (14d)

Brownish orange crystals, yield 86\%. Mp 140-142 ${ }^{\circ} \mathrm{C}$. IR $\left(v, \mathrm{~cm}^{-1}\right)$ : 3429-3125 (OH), $3064(\mathrm{CH}$ aromatic), $2220(\mathrm{CN}), 1604,1488(\mathrm{C}=\mathrm{C}), 1566(\mathrm{C}=\mathrm{N}) .{ }^{1} \mathrm{H}$ NMR (DMSO- $\left.d_{6}\right) \delta 6.03,7.28(2 \mathrm{~s}, 2 \mathrm{H}$, thiazole $\mathrm{H}-2$, pyrimidine $\mathrm{H}-8 \mathrm{a}), 7.33-8.06\left(\mathrm{~m}, 10 \mathrm{H}, 2 \mathrm{C}_{6} \mathrm{H}_{5}\right), 8.40$ (s, 1H, OH). Anal. Calcd. for $\mathrm{C}_{19} \mathrm{H}_{13} \mathrm{~N}_{3} \mathrm{OS}$ (331.39): C, 68.86; H, 3.95; N, 12.68; S, 9.68. Found: C, 68.46; H, 4.35; N, 12.69; S, 10.07 .

5-Hydroxy-7-(4-methoxyphenyl)-3-phenyl-8aH-thiazolo[3,2-a]pyrimidine-6-carbonitrile (14e)

Shiny paige crystals, yield $84 \%$. Mp $309-311^{\circ} \mathrm{C}$. IR $\left(v, \mathrm{~cm}^{-1}\right)$ : 3247-3118 (OH), $3050(\mathrm{CH}$ aromatic), 2838 $\left(\mathrm{CH}_{3}\right), 2200(\mathrm{CN}), 1625,1443(\mathrm{C}=\mathrm{C}), 1532(\mathrm{C}=\mathrm{N}) .{ }^{1} \mathrm{H}$ NMR (DMSO- $\left.d_{6}\right) \delta 3.87\left(\mathrm{~s}, 3 \mathrm{H}, \mathrm{CH}_{3}\right), 6.93,6.96(2 \mathrm{~s}, 2 \mathrm{H}$, thiazole $\mathrm{H}-2$, pyrimidine $\mathrm{H}-8 \mathrm{a}), 7.17-7.37\left(\mathrm{~m}, 9 \mathrm{H}, \mathrm{C}_{6} \mathrm{H}_{4}\right.$, $\left.\mathrm{C}_{6} \mathrm{H}_{5}\right), 9.90(\mathrm{~s}, 1 \mathrm{H}, \mathrm{OH}) .{ }^{13} \mathrm{C}$ NMR (DMSO- $\left.d_{6}\right) \delta 40.3$, $55.1,72.0,102.0,114.3$ (2), 115.0, 121.9, 127.9, 128.5 (2),
128.7 (2), 131.3 (2), 132.0, 133.9, 158.5, 166.0, 167.1. MS $m / z(\%): 363\left[\mathrm{M}^{+}+2\right](1.23), 362\left[\mathrm{M}^{+}+1\right](0.14), 361\left[\mathrm{M}^{+}\right]$ (0.09), 134 (100.00), $77\left[\mathrm{C}_{6} \mathrm{H}_{5}\right]^{+}$(26.84). Anal. Calcd. for $\mathrm{C}_{20} \mathrm{H}_{15} \mathrm{~N}_{3} \mathrm{O}_{2} \mathrm{~S}$ (361.42): C, 66.46; H, 4.18; N, 11.63; S, 8.87. Found: C, 66.27; H, 4.58; N, 11.27; S, 8.47.

7-(4-Chlorophenyl)-5-hydroxy-3-phenyl-8aH-thiazolo[3,2-a]pyrimidine-6-carbonitrile (14f)

Off white crystals, yield $83 \%$. Mp $290-292^{\circ} \mathrm{C}$. IR ( $v$, $\left.\mathrm{cm}^{-1}\right)$ : 3438-3181 (OH), $3080(\mathrm{CH}$ aromatic), $2200(\mathrm{CN})$, 1631, $1483(\mathrm{C}=\mathrm{C}), 1535(\mathrm{C}=\mathrm{N}) .{ }^{1} \mathrm{H}$ NMR (DMSO- $\left.d_{6}\right) \delta$ $7.19,7.21(2 \mathrm{~s}, 2 \mathrm{H}$, thiazole $\mathrm{H}-2$, pyrimidine $\mathrm{H}-8 \mathrm{a}), 7.22-$ $7.95\left(\mathrm{~m}, 9 \mathrm{H}, \mathrm{C}_{6} \mathrm{H}_{4}, \mathrm{C}_{6} \mathrm{H}_{5}\right), 10.01(\mathrm{~s}, 1 \mathrm{H}, \mathrm{OH})$. Anal. Calcd. for $\mathrm{C}_{19} \mathrm{H}_{12} \mathrm{ClN}_{3} \mathrm{OS}$ (365.84): C, 62.38; H, 3.31; N, 11.49; S, 8.76. Found: C, 62.78; H, 3.71; N, 11.12; S, 8.41.

\section{2. 15. Synthesis of $N^{\prime}$-(4-Methoxyphenyl)- $N-(4-$ phenylthiazol-2-yl)formimidamide (15)}

To a solution of 4-phenylthiazol-2-amine (1.76 g, $0.01 \mathrm{~mol})$ in 1,4-dioxane $(20 \mathrm{~mL})$ containing triethylamine $(0.50 \mathrm{ml})$, triethyl orthoformate $(1.48 \mathrm{~g}, 0.01)$ and $p a-$ ra-anisidine $(1.23 \mathrm{~g}, 0.01)$ were added. The reaction mixture was heated under reflux for three hours then poured into a beaker containing ice/water mixture. The formed solid product was collected by filtration and crystallized from 1,4-dioxane.

Pale yellow crystals, yield $92 \%$. Mp $208-210{ }^{\circ} \mathrm{C}$. IR (v, cm $\left.{ }^{-1}\right)$ : 3361-3121 (NH), 3074-3008 ( $\mathrm{CH}$ aromatic), 2950, $2836\left(\mathrm{CH}, \mathrm{CH}_{3}\right), 1606,1462(\mathrm{C}=\mathrm{C}), 1549(\mathrm{C}=\mathrm{N}) .{ }^{1} \mathrm{H}$ NMR (DMSO- $\left.d_{6}\right) \delta 3.79\left(\mathrm{~s}, 3 \mathrm{H}, \mathrm{CH}_{3}\right), 6.87(\mathrm{~s}, 1 \mathrm{H}, \mathrm{CH})$, $6.90(\mathrm{~s}, 1 \mathrm{H}$, thiazole, $\mathrm{H}-5)$, 7.05-7.47 (m, 9H, $\left.\mathrm{C}_{6} \mathrm{H}_{4}, \mathrm{C}_{6} \mathrm{H}_{5}\right)$, $11.47(\mathrm{~s}, 1 \mathrm{H}, \mathrm{NH}) .{ }^{13} \mathrm{C}$ NMR (DMSO- $\left.d_{6}\right) \delta 55.5,114.5$, 114.6, 121.3, 122.1, 126.5 (2), 129.8 (2), 130.3, 131.0, 139.0, $151.2,152.1,157.8,159.0,166.0$. Anal. Calcd. for $\mathrm{C}_{17} \mathrm{H}-$ ${ }_{15} \mathrm{~N}_{3} \mathrm{OS}$ (309.39): C, 66.00; H, 4.89; N, 13.58; S, 10.36. Found: C, 66.37; H, 5.28; N, 13.18; S, 10.59 .

\section{2. 16. General Procedure for the Synthesis of Thiazolo[3,2-a]pyrimidine Derivatives 16a,b}

To a solution of 4-phenylthiazol-2-amine (1.76 g, $0.01 \mathrm{~mol})$ in ethanol $(30 \mathrm{~mL})$ containing a catalytic amount of triethylamine $(0.50 \mathrm{~mL})$ and triethyl orthoformate $(1.48$ g, $0.01 \mathrm{~mol})$, either malononitrile $(0.66 \mathrm{~g}, 0.01 \mathrm{~mol})$ or ethyl cyanoacetate $(1.13 \mathrm{~g}, 0.01 \mathrm{~mol})$ was added. The reaction mixture, in each case, was heated under reflux for five hours then poured into a beaker containing an acidified ice/water mixture. The formed solid product, in each case, was collected by filtration and crystallized from ethanol.

\section{5-Imino-3-phenyl-5H-thiazolo $[3,2-a]$ pyrimidine-6- carbonitrile (16a) \\ Yellow crystals, yield $85 \%$. Mp $190-192{ }^{\circ} \mathrm{C}$. IR ( $v$, $\left.\mathrm{cm}^{-1}\right)$ : 3434-3112 (NH), $3050(\mathrm{CH}$ aromatic), $2210(\mathrm{CN})$,}


1598, $1479(\mathrm{C}=\mathrm{C}), 1523(\mathrm{C}=\mathrm{N}) ;{ }^{1} \mathrm{H}$ NMR $\left(\mathrm{DMSO}-d_{6}\right) \delta$ $6.98(\mathrm{~s}, 1 \mathrm{H}$, thiazole $\mathrm{H}-5), 7.02(\mathrm{~s}, 1 \mathrm{H}$, pyrimidine $\mathrm{H}-7)$, 7.22-7.81 (m, 5H, $\left.\mathrm{C}_{6} \mathrm{H}_{5}\right), 8.60(\mathrm{~s}, 1 \mathrm{H}, \mathrm{NH}) .{ }^{13} \mathrm{C} \mathrm{NMR}$ $\left(\right.$ DMSO- $\left.d_{6}\right) \delta 98.0,101.4,115.0,125.5,127.1(2), 128.4$ (2), 134.9, 149.8, 156.0, 158.0, 168.2. MS m/z (\%): 253 $\left[\mathrm{M}^{+}+1\right](0.09), 252\left[\mathrm{M}^{+}\right](0.09), 176(100.00), 77\left[\mathrm{C}_{6} \mathrm{H}_{5}\right]^{+}$ (13.42). Anal. Calcd. for $\mathrm{C}_{13} \mathrm{H}_{8} \mathrm{~N}_{4} \mathrm{~S}$ (252.29): C, 61.89; H, 3.20 ; N, 22.21; S, 12.71. Found: C, 61.49; H, 3.59; N, 21.81; $\mathrm{S}, 12.31$.

\section{5-Oxo-3-phenyl-5H-thiazolo $[3,2-a]$ pyrimidine-6-car- bonitrile (16b)}

Pale yellow crystals, yield $84 \%$. Mp $170-172{ }^{\circ} \mathrm{C}$. IR $\left(v, \mathrm{~cm}^{-1}\right): 3113(\mathrm{CH}$ aromatic), $2200(\mathrm{CN}), 1689(\mathrm{C}=\mathrm{O})$, 1597, $1482(\mathrm{C}=\mathrm{C}), 1519(\mathrm{C}=\mathrm{N}) .{ }^{1} \mathrm{H}$ NMR $\left(\right.$ DMSO- $\left.d_{6}\right) \delta$ $6.99(\mathrm{~s}, 1 \mathrm{H}$, thiazole $\mathrm{H}-5), 7.01$ (s, $1 \mathrm{H}$, pyrimidine $\mathrm{H}-7)$, 7.23-7.81 (m, 5H, $\left.\mathrm{C}_{6} \mathrm{H}_{5}\right) .{ }^{13} \mathrm{C}$ NMR (DMSO- $\left.d_{6}\right) \delta 98.1$, 101.5, 115.0, 125.5, 127.2 (2), 128.5 (2), 134.8, 149.7, 156.1, 158.2, 168.2. Anal. Calcd. for $\mathrm{C}_{13} \mathrm{H}_{7} \mathrm{~N}_{3} \mathrm{OS}$ (253.28): C, 61.65; H, 2.79; N, 16.59; S, 12.66. Found: C, 61.35; H, 3.01; $\mathrm{N}, 16.30 ; \mathrm{S}, 12.34$.

\section{3. Biology}

Reagents. Fetal bovine serum (FBS) and L-glutamine were obtained from Gibco Invitrogen Company (Scotland, UK). RPMI-1640 medium was provided from Cambrex (New Jersey, USA). Dimethyl sulfoxide (DMSO), doxorubicin, penicillin, streptomycin and sulforhodamine B (SRB) were obtained from Sigma Chemical Company (Saint Louis, MO, USA).

Samples. Stock solutions of compounds $\mathbf{1 - 1 6 b}$ were prepared in DMSO and kept at $-20^{\circ} \mathrm{C}$. Appropriate dilutions of the compounds were freshly prepared just before the assays. Final concentrations of DMSO did not interfere with the cell growth.

Cell cultures. Three human tumor cell lines, MCF-7 (breast adenocarcinoma), NCI-H460 (nonsmall cell lung cancer), and SF-268 (CNS cancer) were used. MCF-7 was obtained from the European Collection of Cell Cultures (ECACC, Salisbury, UK) NCI-H460, SF-268, and normal fibroblast cells (WI 38) were kindly provided by the National Cancer Institute (NCI, Cairo, Egypt). They grew as a monolayer and were routinely maintained in RPMI-1640 medium supplemented with $5 \%$ heat-inactivated FBS, $2 \mathrm{mM}$ glutamine and antibiotics (penicillin $100 \mathrm{U} / \mathrm{mL}$, streptomycin $100 \mu \mathrm{g} / \mathrm{mL}$ ), at $37^{\circ} \mathrm{C}$ in a humidified atmosphere containing $5 \% \mathrm{CO}_{2}$. Exponentially growing cells were obtained by plating $1.5 \times 10^{5}$ cells/mL for MCF- 7 and SF-268 and $0.75 \times 10^{4}$ cells $/ \mathrm{mL}$ for NCI-H460, followed by 24 hours of incubation. The effect of the vehicle solvent (DMSO) on the growth of these cell lines was evaluated in all the experiments by exposing untreated control cells to the maximum concentration $(0.5 \%)$ of DMSO used in each assay.

\section{Results and Discussion}

\section{1. Chemistry}

The 4-phenylthiazol-2-amine was prepared from the reaction of thiourea with $\omega$-bromoacetophenone according to the reported literature. ${ }^{54}$ In the present work, we used the title compound in many heterocyclization reactions followed by studying the cytotoxicity of the resulting compounds against different cancer cell lines. Compound 4-phenylthiazol-2-amine reacted with triethyl orthoformate to produce the ethyl $N$-(4-phenylthiazol-2-yl) formimidate $\mathbf{1}$. The structure of compound $\mathbf{1}$ was based on its analytical and spectral data. The ${ }^{1} \mathrm{H}$ NMR spectrum revealed the presence of a triplet at $\delta 1.91 \mathrm{ppm}$, a quartet at $\delta$ $3.32 \mathrm{ppm}$ for the ethoxy group, a singlet at $\delta 7.28 \mathrm{ppm}$ for thiazole $\mathrm{H}-5$, and a multiplet at $\delta 7.32-7.90 \mathrm{ppm}$ for the $\mathrm{CH}$ group and phenyl moiety. The mass spectrum showed $\left[\mathrm{M}^{+}\right]$at $m / z=232$ in correspondence to the molecular formula $\mathrm{C}_{12} \mathrm{H}_{12} \mathrm{~N}_{2} \mathrm{OS}$.

Due to the excellent yield of compound $\mathbf{1}$, the current study investigated its reactivity with a variety of chemical reagents. Compound 1 reacted with hydrazine hydrate or phenylhydrazine to give the hydrazide derivatives $\mathbf{2 a}$ or $2 \mathbf{b}$, respectively. Moreover, it reacted with aniline to give the $N$-phenyl- $N$ '-(4-phenylthiazol-2-yl)formimidamide 3. Also, it reacted with malononitrile in 1,4-dioxane containing a catalytic amount of triethylamine to give the 2-((4-phenylthiazol-2-ylimino)methyl)malononitrile $\quad 4$ (Scheme 1). Compound 4 was earlier prepared in literature by Covington et al. through the two reported patents. ${ }^{42,43}$ The analytical and spectral data of compound $\mathbf{4}$ were consistent with its structure. Thus, in its mass spectrum, the existing $\left[\mathrm{M}^{+}+2\right]$ ion $(m / z=254),\left[\mathrm{M}^{+}+1\right]$ ion $(\mathrm{m} / z=253)$ and $\left[\mathrm{M}^{+}\right]$ions $(\mathrm{m} / z=252)$ confirmed its molecular weight and structure.

The 4-phenylthiazol-2-amine reacted with phenyl isothiocyanate to give the thiourea derivative 5. Compound $\mathbf{5}$ was previously reported in the literature ${ }^{44,45}$ by Bhargava et al., despite using other reaction conditions involving benzene and heating on a water bath for six hours. ${ }^{44}$ In addition, it reacted with chloroacetylchloride to give the 2-chloro- $N$-(4-phenylthiazol-2-yl)acetamide 6 . The structure of compound $\mathbf{6}$ was established based on its analytical and spectral data. It is worth mentioning that compound 6 was previously synthesized ${ }^{46-50}$ using other reaction conditions. However, the current method was the simplest due to the short reaction time and easily available reagents. Moreover, 4-phenylthiazol-2-amine reacted with ethyl cyanoacetate in dimethylformamide solution to give the 2-cyano- $N$-(4-phenylthiazol-2-yl)acetamide 7. The analytical and spectral data of the latter compound were in agreement with its proposed structure. The ${ }^{1} \mathrm{H}$ NMR spectrum revealed a singlet at $\delta 4.07 \mathrm{ppm}$ for $\mathrm{CH}_{2}$ group, a multiplet at $\delta 7.30-7.90 \mathrm{ppm}$ for benzene ring, a singlet at $\delta 7.91 \mathrm{ppm}$ for thiazole $\mathrm{H}-5$, and a singlet at $\delta 12.39 \mathrm{ppm}$ for the presence of $\mathrm{NH}$ group. 
On the other hand, compound 7 reacted with acetophenone, in the presence of ammonium acetate, to give 6-(1-phenylethylidene)- $5 \mathrm{H}$-thiazolo[3,2-a]pyrimidine derivative 8. Its structure was proven based on its analytical and spectral data. Compound 7 was capable of Gewald's thiophene synthesis. Its one-pot reaction with elemental sulfur and ethyl cyanoacetate in 1,4-dioxane containing a catalytic amount of triethylamine gave the 5-((4-phenylthiazol-2-yl)carbamoyl)thiophene derivative 9. The analytical and spectral data of the latter compound were in agreement with its proposed structure. The ${ }^{1} \mathrm{H}$ NMR spectrum revealed a triplet at $\delta 1.20 \mathrm{ppm}$ for $\mathrm{CH}_{3}$ group, a quartet at $\delta 4.05 \mathrm{ppm}$ for $\mathrm{CH}_{2}$ group, a singlet at $\delta 7.30 \mathrm{ppm}$ for thiazole $\mathrm{CH}-5$, a multiplet at $\delta$ $7.32-7.91 \mathrm{ppm}$ for phenyl moiety, a singlet at $\delta 8.52 \mathrm{ppm}$ for two $\mathrm{NH}_{2}$ groups, and a singlet at $\delta 12.32 \mathrm{ppm}$ due to the presence of $\mathrm{NH}$ group.

The appearance of two $\mathrm{C}=\mathrm{O}$ stretching bands at about 1756 and $1687 \mathrm{~cm}^{-1}$ and the presence of $\mathrm{NH}$ and two $\mathrm{NH}_{2}$ bands at a range of $3427-3164 \mathrm{~cm}^{-1}$ in the IR spectrum proved the proposed structure. Moreover, the mass spectrum of compound 9 showed a molecular ion peak at $m / z=388\left[\mathrm{M}^{+}\right]$corresponding to the molecular formula $\mathrm{C}_{17} \mathrm{H}_{16} \mathrm{~N}_{4} \mathrm{O}_{3} \mathrm{~S}_{2}$.

Compound 7 reacted with malononitrile in the presence of 1,4-dioxane and a catalytic amount of triethylamine to give the thiazol-2-yl-1,2-dihydropyridine derivative 10. On the other hand, the reaction of compound 7 with salicylaldehyde in a 1,4-dioxane solution containing a catalytic amount of piperidine gave the 2-oxo-chromene derivative 11, as outlined in Scheme 2. Compound 11 was previously synthesized by Prashanth et al. and Bondock et al., respectively. ${ }^{51,5}$

The 4-phenylthiazol-2-amine reacted with either cyclopentanone or cyclohexanone in 1,4-dioxane containing a catalytic amount of piperidine to give the condensed products $\mathbf{1 2} \mathbf{a}$ and $\mathbf{1 2 b}$, respectively. In addition, it reacted with acetophenone in an ethanol solution containing a catalytic amount of triethylamine to give compound $\mathbf{1 3}$. Compound 13 was reported previously by Xiaodong et al..$^{53}$ The analytical and spectral data of compounds 12a, $\mathbf{1 2 b}$, and 13 agreed with their respective structures.<smiles>NC(=S)[PH2+]C(=O)CBr</smiles><smiles>Nc1nc(-c2ccccc2)cs1</smiles><smiles>CCO/C=N/c1nc(-c2ccccc2)cs1</smiles>
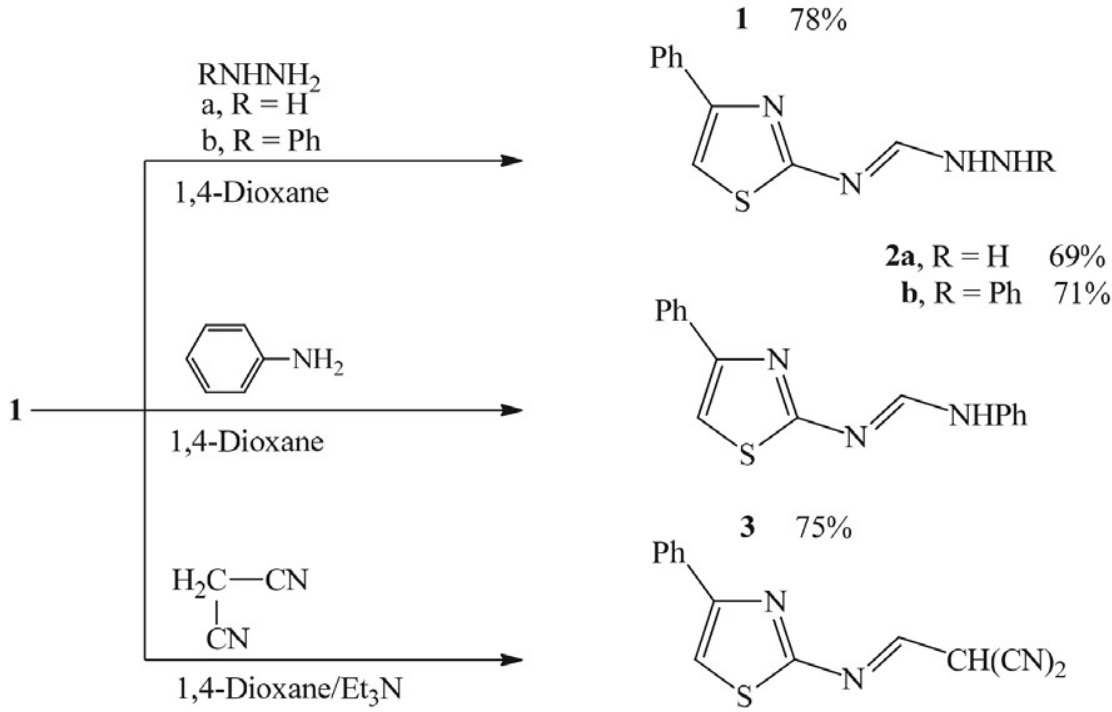

Scheme 1. Synthesis of thiazole derivatives 1, 2a,b, 3 and 4.

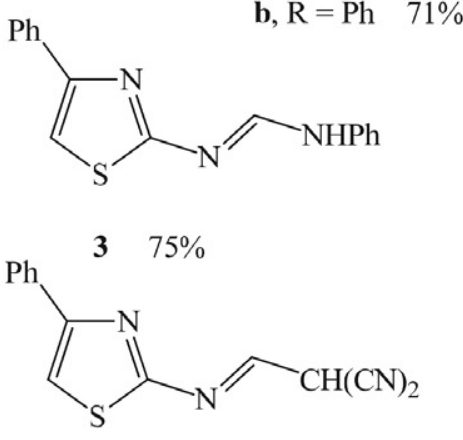


<smiles>Nc1nc(-c2ccccc2)cs1</smiles>

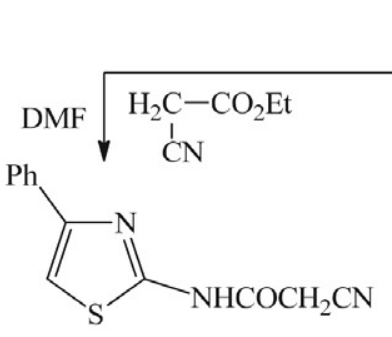

$7 \quad 75 \%$

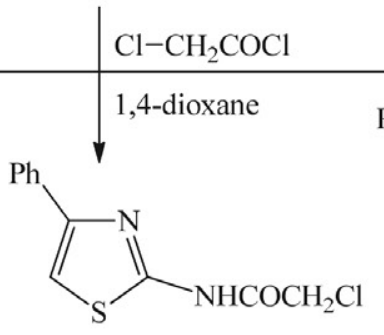

$6 \quad 71 \%$

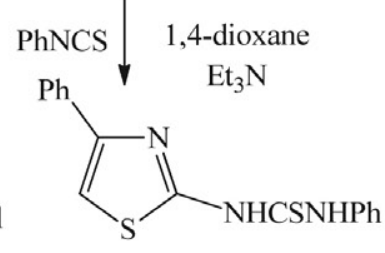

$564 \%$<smiles>CCOC(=O)c1c(N)sc(C(=O)Nc2nc(-c3ccccc3)cs2)c1N</smiles>

Scheme 2. Synthesis of thiazole derivatives $5,6,7,9,11$, thiazolo pyrimidine 8 and thiazol-2-yl pyridine 10 derivatives.

Next, we studied the multi-component reaction starting with compound 4-phenylthiazol-2-amine with the aromatic benzaldehydes and active methylene reagents. Then, the thiazolo[3,2-a]pyrimidines $\mathbf{1 4 a - f}$ were synthesized by the reaction of compound 4-phenylthiazol-2-amine with either benzaldehyde, para-methoxybenzaldehyde, or para-chlorobenzaldehyde and malononitrile or ethyl cyanoacetate in ethanol and triethylamine, respectively (Scheme 3). 

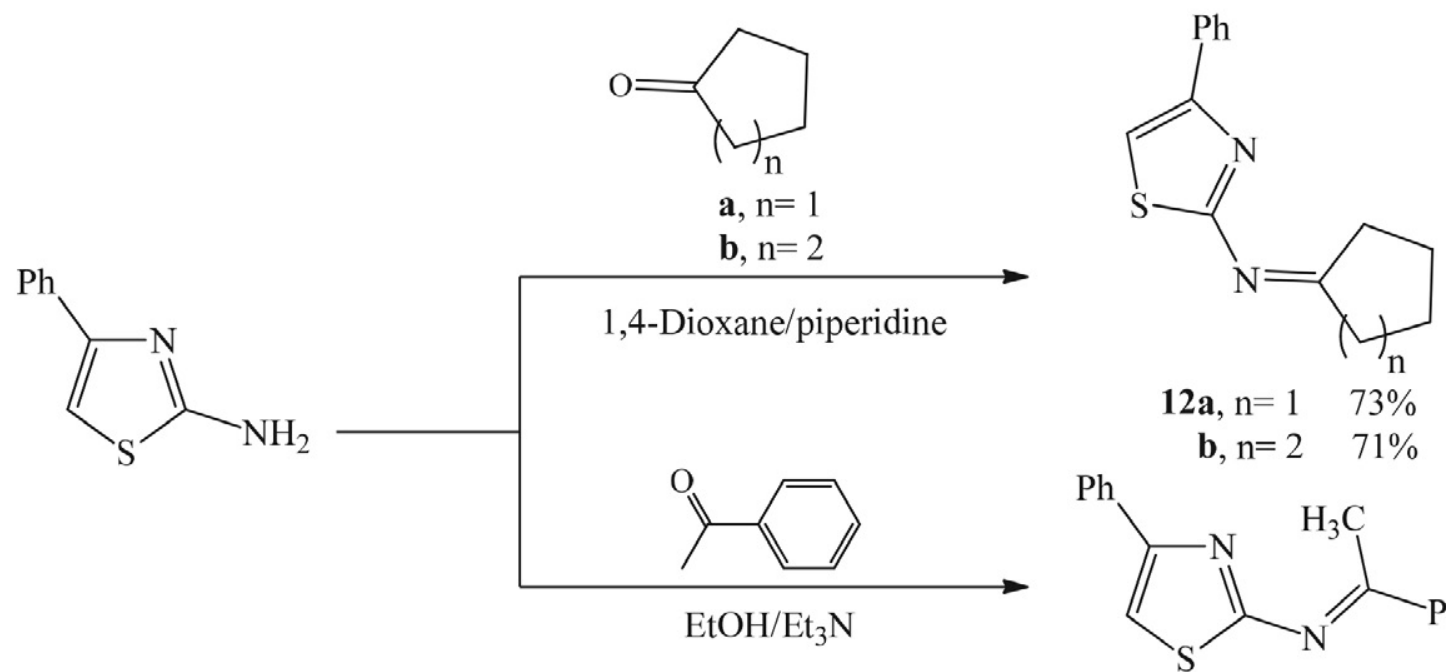

$$
\begin{array}{rr}
\mathbf{1 2} \mathbf{a}, \mathrm{n}=1 & 73 \% \\
\mathbf{b}, \mathrm{n}=2 & 71 \%
\end{array}
$$<smiles>C/C(=N\c1nc(-c2ccccc2)cs1)c1ccccc1</smiles>

$1369 \%$

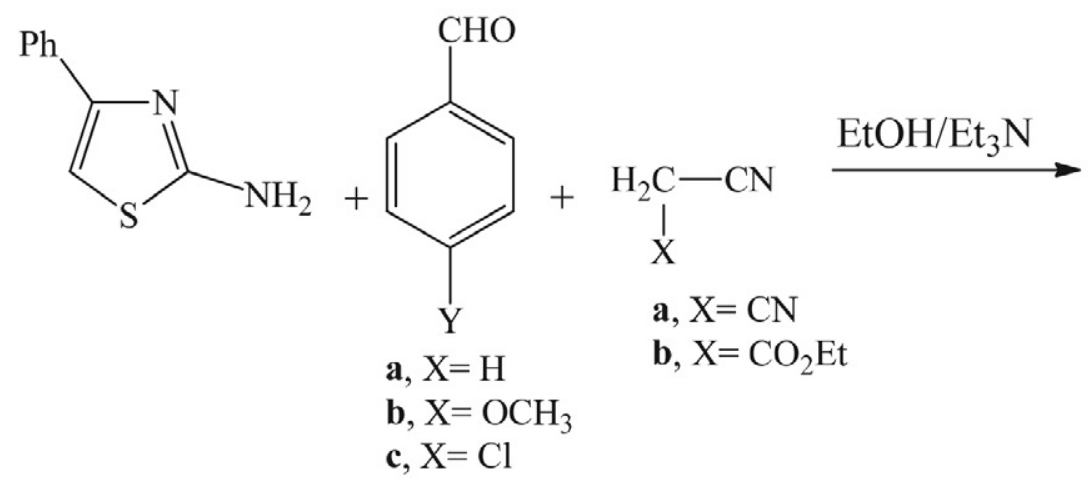

Scheme 3. Synthesis of thiazole derivatives 12a,b, 13 and thiazolo pyrimidine derivatives 14a-f.

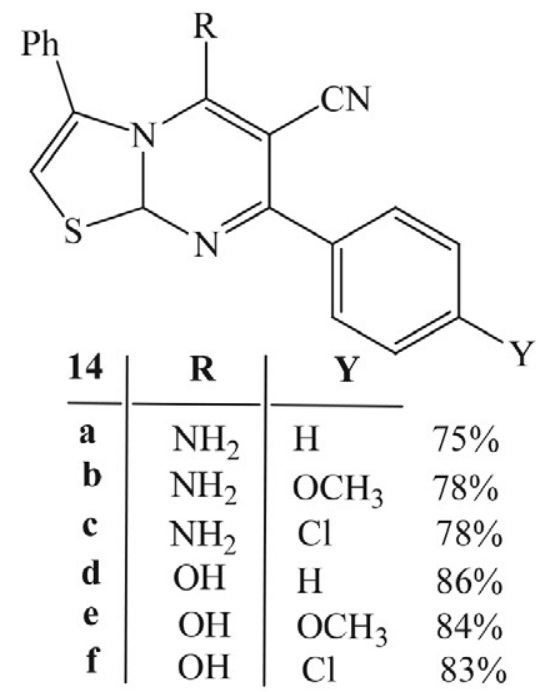

The analytical and spectral data of the latter products were consistent with their respective structures. The ${ }^{1} \mathrm{H}$ NMR spectrum of $14 \mathbf{a}$ as an example revealed a singlet at $d 7.59 \mathrm{ppm}$ for thiazole $\mathrm{H}-2$, a singlet at $d 7.60 \mathrm{ppm}$ for pyrimidine $\mathrm{H}-8 \mathrm{a}$, a multiplet at $d 7.62-7.98 \mathrm{ppm}$ for two phenyl groups and a singlet at $d 8.53 \mathrm{ppm}$ for $\mathrm{NH}_{2}$ group.

In Scheme 4, the reaction of the 4-phenylthiazol-2-amine with triethyl orthoformate and para-anisidine in 1,4-dioxane gave the $N^{\prime}$-(4-methoxyphenyl)- $N$-(4phenylthiazol-2-yl)formimidamide 15, the structure of which was confirmed based on the analytical and spectral data. Finally, the 4-phenylthiazol-2-amine reacted with either malononitrile or ethyl cyanoacetate and triethyl orthoformate in ethanol and triethylamine to form the thiazolo[3,2-a]pyrimidine derivatives $\mathbf{1 6} \mathbf{a}$ and $\mathbf{1 6} \mathbf{b}$, respectively.

\section{2. Biological Activity Evaluations}

\section{2. 1. In Vitro Anticancer Evaluation of the Synthesized Compounds}

The newly synthesized thiazole systems (20 compounds in total) were assessed in vitro for their ability to suppress tumor cell growth ${ }^{55,56}$ on three human tumor cell lines, namely, MCF-7 (breast adenocarcinoma), NCI-H460 (non-small cell lung cancer), and SF-268 (CNS cancer), and normal fibroblasts cells (WI38) after continuous exposure for 48 hours. In addition, the results were compared to the antiproliferative effects of the reference control doxorubicin. ${ }^{57}$ All compounds were dissolved in DMSO at $1 \mathrm{mg} / \mathrm{mL}$ immediately before use and diluted just before being added to the cell culture.

The data in Table 1 represent mean values \pm S.E.M. of three independent experiments performed in dupli- 


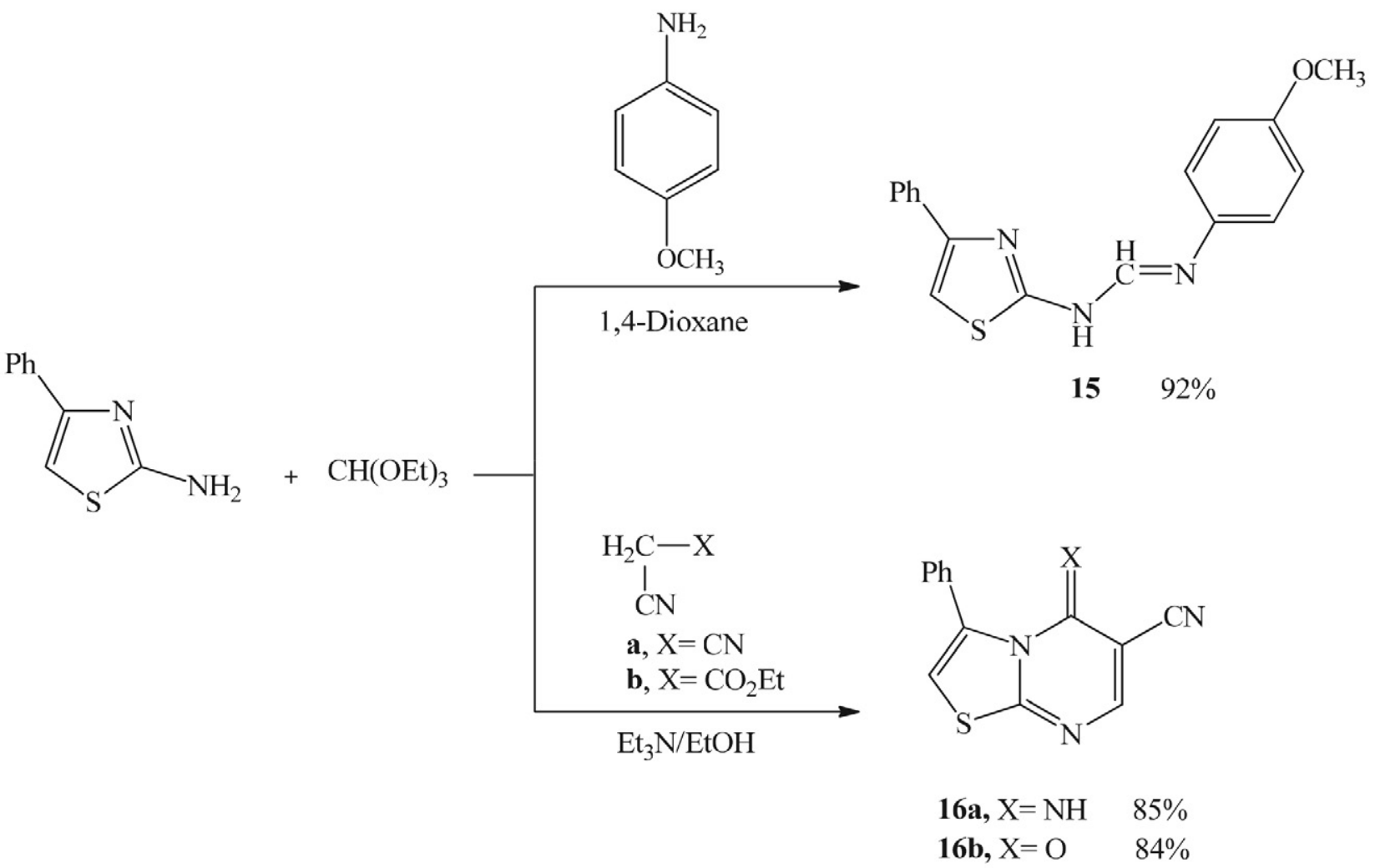

Scheme 4. Synthesis of thiazole derivative 15 and thiazolo pyrimidine derivatives $16 \mathbf{a}$ and $\mathbf{1 6 b}$.

Table 1. Effect of the synthesized compounds in $\mathrm{IC}_{50}(\mu \mathrm{M})$ on the growth of three human tumor cell lines and normal human cell line

\begin{tabular}{ccccc}
\hline Compound No. & & \multicolumn{1}{c}{ IC $_{\mathbf{5 0}} \pm$ S.E.M. $(\boldsymbol{\mu M})^{(\boldsymbol{a})}$} & \\
\hline \multicolumn{1}{c}{ MCF-7 } & NCI-H460 & SF-268 & WI-38 \\
\hline $\mathbf{2 a}$ & $22.40 \pm 2.12$ & $10.42 \pm 3.01$ & $8.63 \pm 1.80$ & $>100$ \\
$\mathbf{2 b}$ & $1.80 \pm 1.00$ & $2.80 \pm 0.30$ & $2.80 \pm 4.20$ & $56.80 \pm 4.0$ \\
$\mathbf{5}$ & $42.60 \pm 2.60$ & $26.60 \pm 2.60$ & $35.20 \pm 12.80$ & $10.50 \pm 5.10$ \\
$\mathbf{6}$ & $32.70 \pm 6.20$ & $28.50 \pm 4.40$ & $40.50 \pm 6.90$ & $70.00 \pm 16.40$ \\
$\mathbf{7}$ & $2.60 \pm 0.20$ & $1.00 \pm 0.60$ & $0.60 \pm 0.08$ & $0.20 \pm 0.01$ \\
$\mathbf{8}$ & $0.20 \pm 0.008$ & $0.03 \pm 0.006$ & $0.05 \pm 0.01$ & $>100$ \\
$\mathbf{9}$ & $0.02 \pm 0.002$ & $0.01 \pm 0.002$ & $0.06 \pm 0.008$ & $>100$ \\
$\mathbf{1 0}$ & $37.00 \pm 7.30$ & $16.70 \pm 2.30$ & $38.40 \pm 2.60$ & $30.60 \pm 6.20$ \\
$\mathbf{1 1}$ & $12.80 \pm 1.40$ & $22.50 \pm 0.40$ & $49.80 \pm 8.60$ & $30.00 \pm 2.30$ \\
$\mathbf{1 2 a}$ & $24.20 \pm 2.40$ & $20.60 \pm 2.80$ & $16.80 \pm 8.50$ & $32.20 \pm 4.60$ \\
$\mathbf{1 2 b}$ & $28.40 \pm 8.80$ & $20.70 \pm 6.20$ & $34.40 \pm 2.40$ & $30.60 \pm 3.00$ \\
$\mathbf{1 3}$ & $22.10 \pm 10.40$ & $30.80 \pm 10.80$ & $26.10 \pm 2.80$ & $28.20 \pm 0.80$ \\
$\mathbf{1 4 a}$ & $0.01 \pm 0.001$ & $0.02 \pm 0.006$ & $0.02 \pm 0.008$ & $>100$ \\
$\mathbf{1 4 b}$ & $14.00 \pm 1.40$ & $22.80 \pm 0.30$ & $22.30 \pm 0.80$ & $32.40 \pm 0.60$ \\
$\mathbf{1 4 c}$ & $0.60 \pm 0.01$ & $0.60 \pm 0.06$ & $0.40 \pm 0.06$ & $50.40 \pm 11.30$ \\
$\mathbf{1 4 d}$ & $33.60 \pm 8.50$ & $40.30 \pm 12.30$ & $30.40 \pm 2.80$ & $62.20 \pm 2.00$ \\
$\mathbf{1 4 e}$ & $0.06 \pm 0.006$ & $0.06 \pm 0.006$ & $0.20 \pm 0.08$ & $40.50 \pm 5.10$ \\
$\mathbf{1 4 f}$ & $30.20 \pm 3.60$ & $38.30 \pm 12.50$ & $42.60 \pm 5.80$ & $58.70 \pm 8.60$ \\
$\mathbf{1 5}$ & $1.20 \pm 0.40$ & $0.80 \pm 0.16$ & $1.30 \pm 0.06$ & $36.40 \pm 1.40$ \\
$\mathbf{1 6 b}$ & $0.80 \pm 0.01$ & $0.03 \pm 0.007$ & $0.60 \pm 0.02$ & $20.20 \pm 3.40$ \\
${ }^{*}$ Doxorubicin & $0.0428 \pm 0.0082$ & $0.0940 \pm 0.0087$ & $0.0940 \pm 0.0070$ & $>100$ \\
\hline
\end{tabular}

${ }^{(a)}$ Drug concentration required to inhibit tumor cell proliferation by $50 \%$ after continuous exposure for 48 hours; data were expressed as means \pm S.E.M. of three independent experiments performed in duplicates.

${ }^{*}$ Doxorubicin was used as a positive control. 


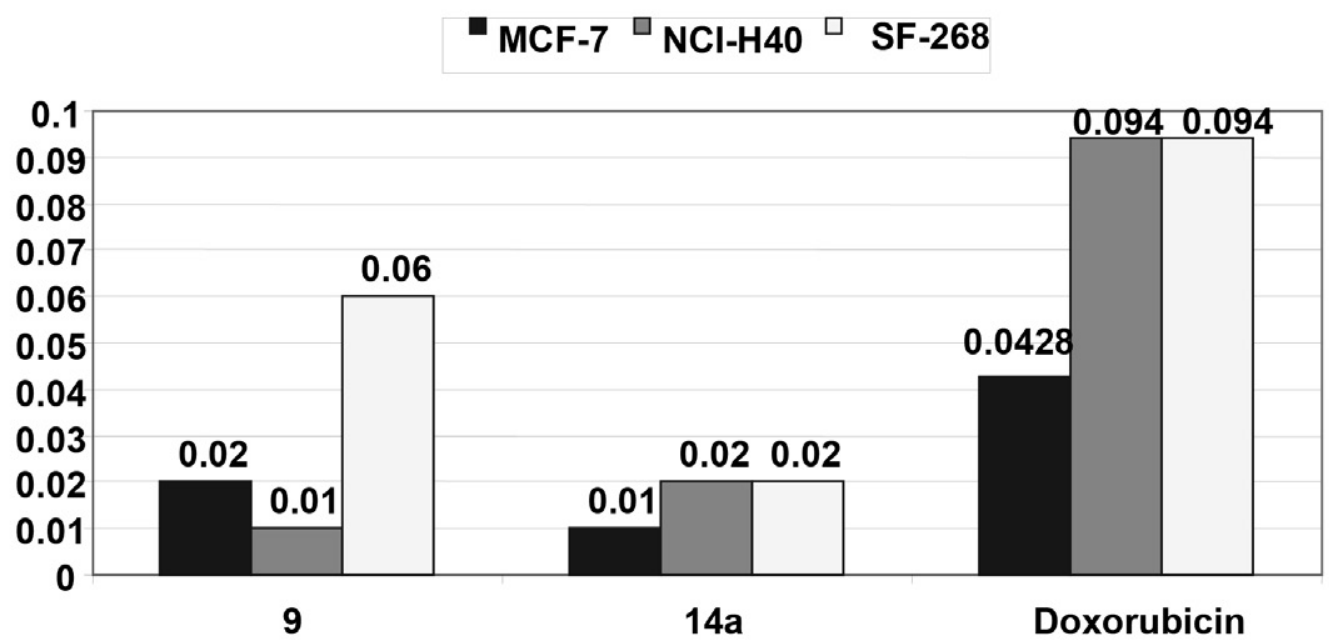

Figure 2. The anticancer evaluation of the most potent synthesized compounds against the three cancer cell lines.

cate. The results indicate that the majority of the compounds demonstrated substantial growth inhibitory effects against the human tumor cells at the concentrations tested.

\section{2. 2. Structure-Activity Relationship}

From Table 1 it is clear that compounds 9 and 14a showed higher effects than the reference doxorubicin for all human cancer cell lines used with $\mathrm{IC}_{50}$ values in the $\mu \mathrm{M}$ range (Figure 2). Although few compounds had low cytotoxicity on a specific tumor cell proliferation, they exhibited significant effects toward the others, such as compound $\mathbf{8}$, which indicated optimal activity compared to the reference used for two cell lines; non-small cell lung cancer (NCI-H460) and SF-268 (CNS cancer). Also, compounds 14e and 16b exhibited a higher effect than the reference doxorubicin on only one cell line (NCI-H460). According to the tested tumor cell, the inhibitory effect of the other compounds towards tumor cell growth varied from high to medium or marginal effects. Moreover, compounds $\mathbf{2 b}$, $7,8,14 c, 14 e, 15$, and $16 b$ exhibited a high effect but not higher than the reference used. For non-small cell lung cancer (NCI-H460), compounds $2 \mathbf{b}, 7, \mathbf{1 4 c}, \mathbf{1 4 e}, \mathbf{1 5}$, and 16b showed a moderate anticancer effect. Also, for SF-268 (CNS cancer), compounds $2 \mathrm{~b}, 7, \mathbf{1 4 c}, 14 \mathrm{e}, 15$, and $\mathbf{1 6 b}$ showed a high activity but not higher than the doxorubicin. On the other hand, compounds $5,6,10,11,12 a, 12 b$, $13,14 \mathrm{~b}, 14 \mathrm{~d}$, and $14 \mathrm{f}$ showed a low potent effect. For normal fibroblast cells (WI38), all compounds showed no cytotoxic effect.

Comparing the cytotoxicity of thiazole derivatives 2a and $\mathbf{2 b}$, it is clear that the cytotoxicity of $\mathbf{2 b}$ is higher than $2 \mathrm{a}$ due to the presence of the phenyl group responsible for the high potency of $\mathbf{2} \mathbf{b}$. Moreover, compound $\mathbf{9}$ showed higher cytotoxicity than doxorubicin due to the presence of the ethoxy group. For some compounds of the thi- azolo[3,2-a]pyrimidine derivatives $\mathbf{1 4 a}-\mathbf{f}$, the presence of the phenyl group such as in compound 14a is responsible for the higher cytotoxicity compared to doxorubicin. The compounds $14 \mathrm{c}$ and $14 \mathrm{e}$ revealed higher cytotoxicity due to the presence of chloro and methoxy groups, respectively. In conclusion, it is clear from the results obtained that the presence of the electronegative phenyl, $\mathrm{Cl}, \mathrm{OCH}_{3}$, and $\mathrm{OC}_{2} \mathrm{H}_{5}$ hydrophobic groups within the thiazole derivatives enhances the cytotoxicity of the tested compounds towards the selected cancer cell lines.

\section{Conclusions}

The objective of the current study was to synthesize a series of thiazole derivatives starting from 4-phenylthiazol-2-amine through its reaction with different chemical reagents. The anticancer activity of some of the newly synthesized compounds (twenty compounds) was evaluated on three human cancer cell lines and a normal human cell line. The results showed that compounds 9 and 14a revealed higher effect than the reference doxorubicin when screened in vitro against the three human cancer cell lines tested, such as MCF-7 (breast adenocarcinoma), NCI-H460 (non-small cell lung cancer), SF-268 (CNS cancer), and normal fibroblasts human cell line (WI-38).

\section{Acknowledgements}

The authors would like to express their gratitude to the Department of Chemistry, Faculty of Science, Cairo University, for conducting this study and providing the necessary facilities.

\section{Conflict of Interest}

The authors declare no conflict of interest. 


\section{References}

1. B. Ghasemi, M. Najimi, Iran. J. Vet. Med. 2016, 10, 47-52. DOI:10.22059/IJVM.2016.57050

2. A. M. Khalil, M. A. Berghot, M. A. Gouda, Eur. J. Med. Chem. 2009, 44, 4434-4440. DOI:10.1016/j.ejmech.2009.06.002

3. S. A. Ouf, S. M. Gomha, M. M. Ewies, I. A. A. Sharawy, J. Heterocycl. Chem. 2018, 55, 258-264. DOI:10.1002/jhet.3040

4. M. M. Ghorab, A. I. El-batal, Boll. Chim. Farm. 2002, 141, 110-117.

5. K. M. Dawood, T. M. A. Eldebss, H. S. A. El-Zahabi, M. H. Yousef, Eur. J. Med. Chem. 2015, 102, 266-276. DOI:10.1016/j.ejmech.2015.08.005

6. N. I. Zelisko, I. L. Demchuk, R. B. Lesyk, Ukr. Biochem. J. 2016, 88, 105-112. DOI:10.15407/ubj88.si01.105

7. O. I. El-Sabbagh, M. M. Baraka, S. M. Ibrahim, C. Pannecouque, G. Andrei, R. Snoeck, J. Balzarini, A. A. Rashad, Eur. J. Med. Chem. 2009, 44, 3746-3753.

DOI:10.1016/j.ejmech.2009.03.038

8. R. N. Sharma, F. P. Xavier, K. K. Vasu, S. C. Chaturvedi, S. S. Pancholi, J. Enzyme Inhib. Med. Chem. 2009, 24, 890-897. DOI: $10.1080 / 14756360802519558$

9. F. A. Hassa, Int. J. Appl. Sci. Technol. 2012, 2, 180-187.

10. I. Kayagil, S. Demirayak, Phosphorus Sulfur Silicon Relat Elem. 2009, 184, 2197-2207. DOI:10.1080/10426500802446181

11. J.-H. Park, M. I. El-Gamal, Y. S. Lee, C.-H. Oh, Eur. J. Med. Chem. 2011, 46, 5769-5777. DOI:10.1016/j.ejmech.2011.08.024

12. T. I. de Santana, M. O. Barbosa, P. A. T. M. Gomes, A. C. N. da Cruz, T. G. da Silva, A. C. L. Leite, Eur. J. Med. Chem., 2018, 144, 874-886. DOI:10.1016/j.ejmech.2017.12.040

13. M. Abdel-Motaal, A. L. Alanzy, M. Asem, Acta Chim. Slov. 2020, 67, 560-569. DOI:10.17344/acsi.2019.5571

14. A. Srinivas, P. Karthik, M. Sunitha, K. V. Reddy, Acta Chim. Slov. 2019, 66, 700-710. DOI:10.17344/acsi.2019.5156

15. M. L. Barreca, J. Balzarini, A. Chimirri, E. De Clercq, L. De Luca, H. D. Höltje, M. Höltje, A. M. Monforte, P. Monforte, C. Pannecouque, A. Rao, M. Zappalà, J. Med. Chem. 2002, 45, 5410-5413. DOI:10.1021/jm020977

16. Y. A. Al-Soud, H. H. Al-Sa'doni, S. O. W. Saber, R. H. M. Al-Shaneek, N. A. Al-Masoudi, R. Loddo, P. La Collac, Z. Naturforsch. 2010, 65b, 1372-1380.

DOI:10.1515/znb-2010-1113

17. M. Madni, S. Hameed, M. N. Ahmed, M. N. Tahir, A. Najim, N. A. Al-Masoudi, C. Pannecouque, Med. Chem. Res. 2017, 26, 2653-2665. DOI:10.1007/s00044-017-1963-1

18. V. Jaishree, N. Ramdas, J. Sachin, B. Ramesh, J. Saudi Chem. Soc. 2012, 16, 371-376. DOI:10.1016/j.jscs.2011.02.007

19. M. Djukic, M. Fesatidou, I. Xenikakis, A. Athina Geronikaki, V. T. Angelova, V. Savic, M. Pasic, B. Krilovic, D. Djukic, B. Gobeljic, M. Pavlica, A. Djuric, I. Stanojevic, D. Vojvodic, L. Saso, Chem-Biol Interact. 2018, 286, 119-131.

DOI:10.1016/j.cbi.2018.03.013

20. G. Saravanan, V. Alagarsamy, C. R. Prakash, P. D. Kumar, T. P. Selvam, Asian J. Res. Pharm. Sci. 2011, 1, 134-138.

21. A. H. Abdelazeem, M. T. El-Saadi, A. G. Safi El-Din, H. A.
Omar, S. M. El-Moghazy, Bioorg. Med. Chem. 2017, 25, 665676. DOI:10.1016/j.bmc.2016.11.037

22. P. S. Banerjee, P. K. Sharma, Med. Chem. Res. 2012, 21, $1491-$ 1508. DOI:10.1007/s00044-011-9615-3

23. B. P. Mallikarjuna, B. S. Sastry, G. V. S. Kumar, Y Rajendraprasad, S. M. Chandrashekar, K. Sathisha, Eur. J. Med. Chem. 2009, 44, 4739-4746. DOI:10.1016/j.ejmech.2009.06.008

24. R. Robinson, J. Chem. Soc. Trans. 1909, 95, 2167-2174. DOI:10.1039/CT9099502167

25. S. Gabriel, Ber. Dtsch. Chem. Ges. 1910, 43, 134-138. DOI:10.1002/cber.19100430117

26. S. Gabriel, Ber. Dtsch. Chem. Ges. 1910, 43, 1283-1287. DOI:10.1002/cber.19100430219

27. A. H. Cook, I. Heilbron, A. L. Levy, J. Chem. Soc. 1947, 15941598. DOI:10.1039/jr9470001594

28. L. F. Frey, K. M. Marcantonio, C.-Y. Chen, D. J. Wallace, J. A. Murry, L. Tan, W. Chen, U. H. Dolling, E. J. J. Grabowski, Tetrahedron 2003, 59, 6363-6373. DOI:10.1016/S0040-4020(03)00878-0

29. W. K. Warburton, Chem. Rev. 1957, 57, 1011-1020. DOI:10.1021/cr50017a004

30. M. Kriek, F. Martins, R. Leonardi, S. A. Fairhurst, D. J. Lowe, P. L. Roach, J. Biol. Chem. 2007, 282, 17413-17423. DOI:10.1074/jbc.M700782200

31. R. M. Mohareb, A. E. M. Abdallah, M. A. Abdelaziz, Med. Chem. Res. 2014, 23, 564-579. DOI:10.1007/s00044-013-0664-7

32. R. M. Mohareb, A. E. M. Abdallah, E. A. Ahmed, Acta Pharm. 2017, 67, 495-510. DOI:10.1515/acph-2017-0040

33. R. M. Mohareb, A. E. M. Abdallah, A. A. Mohamed, Chem. Pharm. Bull. 2018, 66, 309-318. DOI:10.1248/cpb.c17-00922

34. R. M. Mohareb, E. M. Khalil, A. E. Mayhoub, A. E. M. Abdallah, J. Heterocycl. Chem. 2020, 57, 1330-1343. DOI:10.1002/jhet.3870

35. A. E. M. Abdallah, R. M. Mohareb, E. M. Khalil, M. A. M. A. Elshamy, Chem. Pharm. Bull. 2017, 65, 469-477. DOI:10.1248/cpb.c16-00925

36. A. E. M. Abdallah, G. H. Elgemeie, Drug. Des. Devel. Ther. 2018, 12, 1785-1798. DOI:10.2147/DDDT.S159310

37. A. E. M. Abdallah, R. M. Mohareb, E. A. Ahmed, J. Heterocycl. Chem. 2019, 56, 3017-3029. DOI:10.1002/jhet.3697

38. A. E. M. Abdallah, R. M. Mohareb, Pigm. Resin Technol. 2019, 48, 89-103. DOI:10.1108/PRT-11-2017-0085

39. R. M. Mohareb, Y. R. Milad, A. A. Masoud, Acta Chim. Slov. 2021, 68, 72-87. DOI:10.17344/acsi.2020.6182

40. R. M. Mohareb, R. A. Ibrahim, E. S. Alwan, Acta Chim. Slov. 2021, 68, 51-64. DOI:10.17344/acsi.2020.6090

41. R. M. Mohareb, F. M. Manhi, A. Abdelwahab, Acta Chim. Slov. 2020, 67, 83-95. DOI:10.17344/acsi.2019.5224

42. R. R. Covington, D. L. Temple, J. P. Yevich, Pat. Specif. (Aust.) 1985, AU 541789 B2 19850117.

43. R. R. Covington, D. L. J. Temple, J. P. Yevich, Ger. Offen. 1979, DE 2918085 A1 19791115.

44. P. N. Bhargava, S. C. Sharma, Bull. Chem. Soc. Jap. 1965, 38, 905-909. DOI:10.1246/bcsj.38.905

45. T. E. Achary, J. Indian Chem. Soc. 1975, 52, 1204-1206. 
46. S. K. Sonwane, S. D. Srivastava, S. K. Srivastava, Indian J. Chem. 2008, 47B, 633-636.

47. S. K. Sonwane, Proc. Natl. Acad. Sci., India, Section A: Phys. Sci. 2008, 78, 129-136.

48. M. A. El-Maghraby, Indian J. Chem. 1974, 12, 1058-1059.

49. O. Tetu, Bull. Soc. Chim. Fr. 1966, 1, 342-345.

50. B. Dash, J. Inst. Chem. (India) 1979, 51, 151-155. DOI:10.1016/0022-2860(79)80287-2

51. T. Prashanth, B. R. V. Avin, P. Thirusangu; V. L. Ranganatha, B. T. Prabhakar, J. N. N. S.Chandra, S. A. Khanum, Biomed. Pharmacother. 2019, 112, 108707.

DOI:10.1016/j.biopha.2019.108707

52. S. Bondock, A. E.-G. Tarhoni, A. A. Fadda, J. Heterocycl. Chem. 2014, 51, 249-255. DOI:10.1002/jhet.1677
53. X. Tang, J. Yang, Z. Zhu, M. Zheng, W. Wu, H. Jiang, J. Org. Chem. 2016, 81, 11461-11466.

DOI:10.1021/acs.joc.6b02124

54. S. R. Pattan, A. M. Shamrez, J. S. Pattan, S. S. Purohit, V. V. K. Reddy, B. R. Nataraj, Indian J. Chem. 2006, 45B, 1929-1932.

55. A. Monks, D. Scudiero, P. Skehan, R. Shoemaker, K. Paull, D. Vistica, C. Hose, J. Langley, P. Cronise, A. Vaigro-Wolff, M. Gray-Goodrich, H. Campbell, J. Mayo, M. Boyd, J. Natl. Cancer Inst. 1991, 83, 757-766. DOI:10.1093/jnci/83.11.757

56. K. D. Paull, R. H. Shoemaker, L. Hodes, A. Monks, D. A. Scudiero, L. Rubinstein, J. Plowman, M. R. Boyd, J. Natl. Cancer Inst. 1989, 81, 1088-1092. DOI:10.1093/jnci/81.14.1088

57. L. H. Li, F.-L.Yu, Biochem. Mol. Biol. Int. 1993, 31, 879887.

\section{Povzetek}

Načrtovanje in sinteza mnogih novih derivatov tiazola izhaja iz 4-feniltiazol-2-amina, zato smo raziskali reaktivnost te spojine $\mathrm{z}$ različnimi kemičnimi reagenti. Strukture novih spojin smo ugotovili na osnovi elementnih analiz in spektroskopskih podatkov. V nadaljevanju smo za dvajset spojin, ki smo jih sintetizirali, ugotovili opazno (v $\mu \mathrm{M}$ območju) protirakavo delovanje na tri različne človeške rakaste celične linije [MCF-7 (adenokarcinom dojke), NCI-H460 (nedrobnocelični pljučni rak) in SF-268 (CNS rak)] ter na celično linijo normalnih človeških fibroblastov (WI-38). Rezultati so pokazali, da sta spojini 9 in 14a bolj učinkoviti kot pa referenčna spojina doksorubicin.

Except when otherwise noted, articles in this journal are published under the terms and conditions of the Creative Commons Attribution 4.0 International License 